\title{
Regularity for Fully Nonlinear P-Laplacian Parabolic Systems: the Degenerate Case
}

\author{
Dung LE1 1
}

\begin{abstract}
This paper studies Hölder regularity property of bounded weak solutions to a class of strongly coupled degenerate parabolic systems.
\end{abstract}

\section{Introduction}

We study the Hölder regularity of bounded weak solutions of nonlinear $p$-Laplacian parabolic systems of the form

$$
u_{t}=\operatorname{div}(A(u, D u))+F(u, D u),
$$

in a domain $Q=\Omega \times(0, T) \subset \mathbb{R}^{n+1}$, with $\Omega$ being an open subset of $\mathbb{R}^{n}, n \geq 1$. The vector valued function $u, f$ take values in $\mathbb{R}^{m}, m \geq 1$. Du denotes the spatial derivative of $u$. Here, $A(u, \zeta)$ is a nonlinear map from $\mathbb{R}^{m} \times \mathbb{R}^{n m}$ into $\mathbb{R}^{n m}$.

A weak solution $u$ to (1.1) is a function $u \in W_{2}^{1,0}\left(Q, \mathbb{R}^{m}\right)$ such that

$$
\iint_{Q}\left[\left\langle-u, \phi_{t}\right\rangle+\langle A(u, D u), D \phi\rangle\right] d z=\iint_{Q}\langle F(u, D u), \phi\rangle d z
$$

for all $\phi \in C_{0}^{1}\left(Q, \mathbb{R}^{m}\right)$. Here, we write $d z=d x d t$.

The evolution $p$-Laplacian scalar equation has been one of most widely studied nonlinear degenerate parabolic equations. The particular feature of (1.1) is its gradient-dependent diffusivity. Such systems, and their stationary counterparts, appear in different models in non-Newtonian fluids, turbulent flows in porous media, certain diffusion or heat transfer processes, and recently in image processing.

A large body of literature on $p$-Laplacian systems has been devoted to the following system

$$
u_{t}=\operatorname{div}\left(|D u|^{p-2} D u\right)+F(u, D u)
$$

which is a special case of (1.1) where $A(u, D u)=|D u|^{p-2} D u$ does not depend explicitly on $u$. In this case, the regularity theory of bounded weak solutions was then almost settled and masterfully presented in the text book [1] (see also [4] for the stationary counterpart). The techniques and results also hold for systems where $A$ depends smoothly on $x, t$. In fact, under suitable assumptions on $F$, we now know that bounded weak solutions to the above systems has Hölder continuous spatial derivatives. The theory was then based on a far-reaching combination of generalized DiGiorgi and Moser's methods for scalar equations.

\footnotetext{
${ }^{1}$ Department of Mathematics, University of Texas at San Antonio, One UTSA Circle, San Antonio, TX 78249. Email: dle@math.utsa.edu

Mathematics Subject Classifications: 35K65, 35B65.

Key words: Parabolic systems, Degenerate systems, Partial Hölder regularity.
} 
However, this method breaks down in dealing with systems (1.1) allowing more general structural conditions and with the diffusivity $A$ depending explicitly on the unkown $u$. First of all, the dispersion of the eigenvalues of the derivative of $A$ with respect to the second variable $D u$ will prevent the Moser type iteration techniques in [1, Chapter IX] from being applicable in order to show that $|D u|$ is locally bounded, a starting and crucial point in defining the scaled cylinders in the next steps. Secondly, the presence of $u$ in $A$ will create extra terms when one differentiates the system in order to obtain a new system satisfied by $D u$. These extra terms may not be well defined if $u$ is not yet known to be Hölder continuous.

In this work, we choose a different approach. We will establish the Hölder continuity of $u$ by using a homotopy argument. We assume that the system (1.1) can be imbedded in a family of systems and at least one of which has the property that its bounded weak solutions are Hölder continuous and satisfy a scaling decay estimate. Under suitable assumptions, we show that this property will be carried onto bounded weak solutions of the considered system. This type of decay estimates with scaling was also used in [1] using the local supremum norm of $|D u|$. In our case, since $|D u|$ is not locally bounded and the best we can say is that $|D u|^{q}$ is locally integrable for some $q>p$. The scaled cylinders in this work must then be defined differently. We will use the average mean of $|D u|^{p}$ instead of its unavailable supremum norm.

Thus, we will consider a family of parabolic systems parameterized by $\nu \in[0,1]$

$$
u_{t}=\operatorname{div}(A(\nu, u, D u))+F(\nu, u, D u), \text { in } Q=\Omega \times(0, T) \subset \mathbb{R}^{n+1} .
$$

Assuming (1.1) is the above system when $\nu=1$ and Hölder continuity results are known for the system when $\nu=0$. Inspired by [1, Proposition 3.1], we introduce the so call scaling decay property $\mathrm{D}$ ) which is Hölder continuity for weak solutions to scalar degenerate equations. We then consider a subset $\mathcal{I}$ of parameters in $[0,1]$ where bounded weak solutions of the above system satisfy this property. The main goal is then to prove that $\mathcal{I}$ is both open and closed in $[0,1]$ so that $\mathcal{I}=[0,1]$ and the desired Hölder continuity for solutions to (1.1) is obtained. Our first two main results concerning the open and closed properties of $\mathcal{I}$ will be presented under two sets of conditions as they will be established by using different tools, and they may be independently of interest in other applications.

The main vehicle in the proof of $\mathcal{I}$ being open is the Proposition 4.3 , which is the $p$ Laplacian version of the nonlinear heat approximation result in [12. Basically, it asserts that if a vector valued function $u$ almost and weakly solves a system like (1.2), with $\nu \in I$, then it can be approximated in certain controllable way by a solution $v$ of the system. By this, property $\mathrm{D}$ ) of $v$ can be carried over to $u$. The proof of this $p$-Laplacian approximation version is not a simple extension of the result in [12] as our systems are degenerate (or singular) and many more technical tools. Among them is a measure theoretic result Lemma 3.8 in Section 3 establishing uniform continuity of the integrals of the derivatives of approximated solutions. As a consequence of this, in Lemma 3.11, we also present a result on higher integrability of the derivatives of "almost" weak solutions to a $p$-Laplacian system. Similar results for weak solutions to $p$-Laplacian systems were first reported in [8].

On the other hand, the above argument is local by nature and cannot be used to prove that $I$ is closed as it lacks certain uniform estimates in order to show that limits of a sequence of regular solutions are also regular. To this end, we will use a different approach deriving 
uniform and global estimates for the integrals of spatial derivatives of regular solutions with uniform bounded norms.

In this paper, our main results only concern the degenerate case, i.e. $p>2$. The singular case, $p<2$, can be dealt with in a similar way but much more subtle and will be reported in a forthcoming work. However, most of our main tools work for both cases and we report them here in Section 3 and Section 4 for future references. We will specifically state the range of $p$ for which our results hold.

The paper is organized as follows. In Section 2, we will introduce notations and discuss in details our hypotheses and main theorems. Section 3 collects technical lemmas. Section 4 presents our main vehicles - the $p$-Laplacian nonlinear approximation results. The proof that $I$ is open will be given in Section 5. Finally, Section 6 details the proof of $I$ being closed and concludes our paper.

\section{Main results}

Throughout this paper, for some $z_{0}=\left(x_{0}, t_{0}\right) \in \mathbb{R}^{n+1}$ and $R, \rho>0, Q_{R, \rho}\left(z_{0}\right)$ denotes the parabolic cylinder centered at $z_{0}$ with radius $R, \rho$. That is, $Q_{R, \rho}\left(z_{0}\right)=B_{R}\left(x_{0}\right) \times\left[t_{0}-\rho, t_{0}\right]$. We also abbreviate by $S_{R, \rho}\left(z_{0}\right)=B_{R}\left(x_{0}\right) \times\left\{t_{0}-\rho\right\} \cup \partial B_{R}\left(x_{0}\right) \times\left[t_{0}-\rho, t_{0}\right]$, the parabolic boundary of $Q_{R}\left(z_{0}\right)$. If the center $z_{0}$ was understood, we would simply write $Q_{R, \rho}, S_{R, \rho}$ for $Q_{R, \rho}\left(z_{0}\right)$ and $S_{R, \rho}\left(z_{0}\right)$ respectively.

For a given cylinder $Q=B \times[a, b]$ and $p>1$, we consider the space $V_{p}(Q)=V_{p}\left(Q, \mathbb{R}^{m}\right)$ of vector valued functions $u: Q \rightarrow \mathbb{R}^{m}$ with norm $\|\cdot\|_{V_{p}(Q)}$ defined by

$$
\|u\|_{V_{p}(Q)}=\sup _{t \in[a, b]}\|u(\cdot, t)\|_{L^{2}(B)}+\|D u\|_{L^{p}(Q)} .
$$

By $V_{p}^{0}(Q)$ we denote the closure of $C_{0}^{1}(Q)$ in $V_{p}(Q)$ with respect to the above norm.

Let $z_{0}=\left(x_{0}, t_{0}\right)$ and $Q_{R, \rho}\left(z_{0}\right)$ be any parabolic cylinder in $R^{n+1}$, the following scaled norm will also be used

$$
\|u\|_{V\left(Q_{R, \rho}\right)}=\sup _{t \in\left[t_{0}-\rho, t_{0}\right]}\left(R^{-n} \int_{B_{R}\left(x_{0}\right)} u(x, t) d x\right)^{\frac{1}{2}}+\left(R^{p} R^{-n} \rho^{-1} \iint_{Q_{R, \rho}}|D u|^{p} d z\right)^{\frac{1}{p}} .
$$

Obviously, this norm is invariant via dilations.

For any integrable function $u: Q \rightarrow \mathbb{R}^{m}$ and any measurable subset $A$ of $Q$, we write

$$
u_{A}=\frac{1}{|A|} \int_{A} u(z) d z=\oiiint_{A} u(z) d z
$$

If $A$ is a cylinder $Q_{R, \rho}=Q_{R, \rho}\left(z_{0}\right)$ and there is no possibility of ambiguity, we simply write $u_{R, \rho}=u_{Q_{R, \rho}}$. Furthermore, if $\rho$ is defined in term of $R$ and the relation between $R, \rho$ is clear we also abbreviate $u_{R, \rho}$ by $u_{R}$ for the sake of simplicity.

As our results are local in nature, without loss of generality, we will simply consider $Q$ being the unit parabolic cylinder $B_{1}(0) \times[-1,0]$ throughout this paper. We then consider a family of systems

$$
u_{t}=\operatorname{div}(A(\nu, u, D u))+F(\nu, u, D u), \text { in } Q=B_{1} \times(-1,0) \subset \mathbb{R}^{n+1} \text { and } \nu \in[0,1] .
$$


By a bounded weak solution $u$ to this system we mean a bounded vector valued function $u$ satisfying

$$
\iint_{Q}\left[-u \phi_{t}+\langle A(\nu, u, D u), D \phi\rangle\right] d z-\iint_{Q} F(\nu, u, D u) \phi d z=0, \quad \forall \phi \in C_{0}^{1}\left(Q, \mathbb{R}^{m}\right) .
$$

For simplicity, we will mainly consider the case $F \equiv 0$ in our discussion. The presence of $F$ can be treated with minor modifications and we will briefly discuss this case at the end of this section.

We will always consider matrices $A(\nu, u, \zeta)$ satisfying the following ellipticity condition

E) There are positive constants $\lambda, \Lambda$ such that for all $\zeta, \eta \in \mathbb{R}^{n m}, u \in \mathbb{R}^{m}$

$$
\langle A(\nu, u, \zeta), \zeta\rangle \geq \lambda|\zeta|^{p}, \quad|\langle A(\nu, u, \zeta), \eta\rangle| \leq \Lambda|\zeta|^{p-1}|\eta| .
$$

In the study of the Hölder regularity of a weak solution $u$, it is now well known that (see [3]) one needs to establish a mean oscillation decay estimate: For some $\tau, \alpha \in(0,1)$ and any $Q_{R}=Q_{R, \rho}(x, t) \subset Q$, there are positive constant $\tau_{0}, C\left(\tau_{0}\right)$ such that

$$
\oiiint_{Q_{\tau R, \tau \rho}}\left|u-u_{\tau R, \tau \rho}\right|^{2} d z \leq C\left(\tau_{0}\right) \tau^{\alpha} \oiiint_{Q_{R, \rho}}\left|u-u_{R, \rho}\right|^{2} d z \quad \forall \tau \in\left(0, \tau_{0}\right) .
$$

However, due to the degeneracy/singularity of the diffusion matrix $A$, such decay estimates do not hold in general for cylinders whose space-time configuration are uniform for all solutions and depend only on the parameters defining the systems. Roughly speaking, we can only obtain here (2.3) when $R, \rho$ are linked via an intrinsic scaling determined by the solution $u$ itself. Yet this decay property still gives the desired Hölder continuity. The idea of using scaled cylinders was known in literature (see [1]) where scalar equations were studied so that Harnack type inequalities could be established and gave the Hölder continuity. Here, such techniques are no longer available and we have to scale the cylinders by using the average oscillations of weak solutions. Being inspired by [1, Proposition 3.1], we introduce the following decay property. Note that the mean oscillation of vector valued solutions is used here in place of the essential oscillation for scalar solutions in [1].

We say that a bounded vector valued function $v: Q \rightarrow \mathbb{R}^{m}$ satisfies a scaling decay property if

D) Let $M=\sup _{Q}|v|$. For any $R_{0}>0, \eta \in(0,1)$, and $\left(x_{0}, t_{0}\right) \in Q$ there are positive numbers $A, K, L, \alpha_{0}, \omega_{0}$ depending on $M, \eta$ (with $K, A$ sufficiently large) such that we can define the following sequences

$$
R_{k}=\frac{R_{0}}{K^{k}}, \quad \omega_{k+1}=\max \left\{\eta \omega_{k}, L R_{n}^{\alpha_{0}}\right\}, \quad S_{k}=\frac{\omega_{k}}{A}, Q_{k}=B_{R_{k}}\left(x_{0}\right) \times\left[t_{0}-S_{k}^{2-p} R_{k}^{p}, t_{0}\right] .
$$

such that if

$$
\omega_{0}^{p} \geq \oiiint_{Q_{0}}\left|v-(v)_{0}\right|^{p} d z, \quad(v)_{0}=\oiiint_{Q_{0}} v d z
$$

then for any integer $k=1, \ldots$

$$
\omega_{k}^{p} \geq \oiiint_{Q_{k}}\left|v-(v)_{k}\right|^{p} d z, \quad(v)_{k}=\oiiint_{Q_{k}} v d z .
$$


Our first main result shows that if (2.1), for some $\nu$, is a "nice" system in the sense that its bounded weak solutions satisfying the decay property D), then "near by" systems are also nice. To be more precise, let us describe the this result in details here. We first suppose that the family of systems (2.1) contains at least a "nice" one.

I) There is a nonempty set $\mathcal{I} \subset[0,1]$ such that for any $\nu$ in $I$ the decay property $\mathrm{D}$ ) holds for any bounded weak solution to (2.1). The same assumption applies to the systems with $u$ being replaced by any constant vector $C$, i.e.

$$
\iint_{Q}\left[-u \phi_{t}+\langle A(\nu, C, D u), D \phi\rangle\right] d z-\iint_{Q} F(\nu, C, D u) \phi d z=0, \quad \forall \phi \in C_{0}^{1}\left(Q, \mathbb{R}^{m}\right)
$$

We then consider the following structural assumptions on the matrices $A(\nu, u, D u)$.

O.1) (Uniform ellipticity) For any $\nu \in[0,1], A(\nu, u, \zeta)$ satisfies the ellipticity condition E) for some positive constants $\lambda, \Lambda$. In addition, $\frac{\partial A}{\partial \zeta}(\nu, u, \zeta)$ exists and there is $c_{0}>0$ such that

$$
\left\langle\frac{\partial A}{\partial \zeta}(\nu, u, \zeta), \zeta\right\rangle \geq c_{0}|\zeta|^{p}
$$

for any bounded weak solution $u$ of (2.1).

O.2) (Monotonicity) For any $w \in \mathbb{R}^{m}$ and $U, V \in \mathbb{R}^{n m}$, there holds

$$
\langle A(w, U)-A(w, V), U-V\rangle \geq \lambda_{0}(U, V)|U-V|^{2},
$$

where

$$
\lambda_{0}(U, V)=c_{0} \begin{cases}\min \left\{|U|^{p-2},|V|^{p-2}\right\} & U \neq 0 \text { or } V \neq 0 \\ 0 & \text { otherwise }\end{cases}
$$

for some positive constant $c_{0}$.

O.3) (Continuity) $A(\nu, u, \zeta)$ is Hölder in $\nu \in[0,1]$ and Lipschitz in $u$. That is,

$$
\begin{gathered}
|A(\nu, u, \zeta)-A(\mu, u, \zeta)| \leq C|\nu-\mu|^{\theta}|\zeta|^{p-1} \\
|A(\nu, u, \zeta)-A(\nu, v, \zeta)| \leq C|u-v||\zeta|^{p-1}
\end{gathered}
$$

for some $C, \theta>0$ and any $\nu, \mu \in[0,1], u, v \in \mathbb{R}^{m}, \zeta \in \mathbb{R}^{n m}$.

O.4) (Existence) For $\nu \in \mathcal{I}$ and any cylinder $Q^{\prime} \subset Q$, and any vector valued function $g \in C^{1}\left(Q^{\prime}\right)$, the system

$$
\left\{\begin{array}{l}
\iint_{Q_{R}}\left[-u \phi_{t}+\langle A(\nu, u, D u), D \phi\rangle\right] d z=0, \quad \forall \phi \in C_{0}^{1}\left(Q^{\prime}, \mathbb{R}^{m}\right), \\
u=g \text { on } S_{Q^{\prime}}
\end{array}\right.
$$

has a bounded weak solution $u$ (and thus satisfies the property D)).

O.5) (Uniform maximum principle) For any $\lambda \in[0,1]$ and $Q^{\prime} \subset Q$, if $g \in C^{1}\left(Q^{\prime}\right)$ then there is a constant $C\left(\|g\|_{L^{\infty}\left(Q^{\prime}\right)}\right)$ such that any weak solution $u$ to the system (2.9) is bounded and satisfies the estimate $\|u\|_{L^{\infty}\left(Q^{\prime}\right)} \leq C\left(\|g\|_{L^{\infty}\left(Q^{\prime}\right)}\right)$. 
Remark 2.1 We should remark that if the argument $u$ in $A(\nu, u, D u)$ is replaced by a constant vector and the data $g$ is bounded then the existence of a weak solution in O.4) is granted by classical approximation methods as in [9] or [16]. Otherwise, the existence condition O.4) can be satisfied by using Galërkin's method if $g$ is sufficiently regular and the solution $u$ is known a-priori to be Hölder continuous. Since $\mathcal{I}$ is the set of parameters for which the systems has bounded weak solutions being Hölder continuous, O.4) is justified.

Our first main result then asserts that the set $\mathcal{I}$, where the property D) holds, is open.

Theorem 2.2 Suppose that I) and O.1)-O.5) hold and $p>2$. Then $\mathcal{I}$ is open in the usual topology of $[0,1]$. Moreover, bounded weak solutions to the system (2.1) with $\nu \in \mathcal{I}$ are Hölder continuous.

The above theorem relies on a nontrivial generalization of the so called nonlinear heat approximation lemma which was introduced in our earlier work [12] concerning nondegenerate systems.

Next, we will give conditions for the set $\mathcal{I}$ to be closed in $[0,1]$. To this end, we take a sequence $\left\{\nu_{k}\right\}$ in $\mathcal{I}$ such that $\nu_{k} \rightarrow \mu$ and we will show that $\mu \in \mathcal{I}$. We first require that any bounded weak solution $u$ to (2.1), with $\nu=\mu$, can be weakly approximated by "nice" solutions.

II) For each $\nu \in \mathcal{I}$, the system (2.1) satisfies the existence condition O.4) and maximum principle O.6). Moreover, if $\nu_{k} \subset \mathcal{I}$ and $\nu_{k} \rightarrow \mu$ then for any bounded weak solution $u$ to (2.1) with $\nu=\mu$ there is a sequence $\left\{v_{k}\right\}$ of Hölder continuous solutions to (2.1), with $\nu=\nu_{k}$, such that $D v_{k}$ converges weakly to $D u$ in $L^{p}(Q)$ and the $L^{\infty}$ norms of $v_{k}$ are bounded uniformly in terms of that of $u$.

Although it will be shown in Section 4 that the above assumption holds under O.1)O.5) via nonlinear heat approximation, we state II) here for our next result, which can be of interest in itself, so that it is independent of Theorem 2.2. Apparently, II) could also be verified by other means via weaker assumptions than O.1)-O.5).

We then consider $\nu \in \mathcal{I}$ and a $C^{1}$ solution $v$ to

$$
\begin{cases}v_{t}=\operatorname{div}(A(\nu, v, D v)) & \text { in } Q \\ v=g & \text { on } S .\end{cases}
$$

The boundary condition $g$ is assumed to be smooth. By II), the above system satisfies the maximum principle and we can define

$$
M_{\nu, v}=\sup _{Q_{\frac{3}{4}}}|v|
$$

For any bounded weak solution $v$ to (2.10), we then impose the following assumptions on the structure of the system. 
M.1) The matrix $\left(A_{k l}^{i j}\right)=\frac{\partial A}{\partial \zeta}(\nu, v, \zeta)$ is elliptic with the ellipticity constants $\lambda_{\nu, v}, \Lambda_{\nu, v}$. That is

$$
\sum_{i, j=1}^{m} \sum_{k, l=1}^{n} A_{k l}^{i j} \eta_{k}^{i} \eta_{l}^{j} \geq \lambda_{\nu, v}|\eta|^{2}, \quad \sum_{i, k}\left(\sum_{j, l} A_{k l}^{i j} \eta_{l}^{j}\right)^{2} \leq \Lambda_{\nu, v}^{2}|\eta|^{2} \quad \forall \eta \in \mathbb{R}^{n m} .
$$

Moreover, for some positive constants $\lambda_{\nu}, \Lambda_{\nu}$ we have

$$
\lambda_{\nu, v} \geq \lambda_{\nu}|\zeta|^{p-2}, \quad \Lambda_{\nu, v} \leq \Lambda_{\nu}|\zeta|^{p-2} .
$$

If $n>2$, we also assume that

$$
\sup \left\{\frac{\Lambda_{\nu, v}}{\lambda_{\nu, v}}: \quad v \text { is a bounded weak solution to }(2.10)\right\}<\frac{n}{n-2} .
$$

M.2) For every $\nu$ in $I$ and any bounded weak solution $v$ to (2.10), there exists a positive constant $a_{\nu, v}$ such that

$$
\left|\frac{\partial A}{\partial v}(\nu, v, \xi)\right| \leq a_{\nu, v}|\xi|^{p-1} \text { with } 2 a_{\nu, v} M_{\nu, v}(p+n-1)<\sigma_{0} \widehat{\lambda}_{\nu}
$$

where $\sigma_{0}$ is a fixed number in $(0,1)$ and

$$
\widehat{\lambda}_{\nu}=\left(1-\delta^{2}\right) \lambda_{\nu} \text { and } \delta=\frac{n-2}{n} \sup \left\{\frac{\Lambda_{\nu, v}}{\lambda_{\nu, v}}: \quad v \text { is a bounded weak solution to (2.10) }\right\} \text {. }
$$

Note that $\hat{\lambda}_{\nu, v}>0$ due to (2.13). Meanwhile, (2.13) requires that the principal eigenvalues $\Lambda_{\nu, v}, \lambda_{\nu, v}$ of $\frac{\partial A}{\partial \zeta}$ are not too far apart (when $n>2$ ). We should also remark that the constants $\lambda_{\nu}, \Lambda_{\nu}$ could be allowed to depend on $v$ as long as there were fixed positive numbers $c_{1}, c_{2}$ such that quotient $c_{1} \leq \lambda_{\nu} / \Lambda_{\nu} \leq c_{2}$. We assume however that they are constants for the sake of simplicity.

Our next main result reads

Theorem 2.3 Assume the conditions II) and M.1)- M.2) and that $p>2$. The set $I$ is closed in $[0,1]$.

Combining with the results of the previous theorem, as O.1)-O.5) are sufficient for II), we then have

Theorem 2.4 Assume I), O.1)-O.5) and M.1)-M.2) and $p>2$ then $I=[0,1]$. Thus, bounded weak solutions are Hölder continuous.

Finally, we remark that our proof continues to hold for systems like

$$
u_{t}=\operatorname{div}(A(\nu, u, D u))+F(\nu, u, D u),
$$

if the nonlinearity $F$ satisfies

$$
\left|\frac{\partial F}{\partial v}(\nu, v, \xi)\right| \leq C+C|v|^{l}+\varepsilon_{0}|\xi|^{p}, \quad\left|\frac{\partial F}{\partial \xi}(\nu, v, \xi)\right| \leq C+C|v|^{l}+\varepsilon_{0}|\xi|^{p-1}
$$

for some $l>0$ and sufficiently small $\varepsilon_{0}>0$. The presence of $F$ would cause extra terms in our arguments but they can be easily treated by invoking the Hölder and Young inequalities. 


\section{Technical lemmas}

In this section, we present various estimates on a vector valued function $u$ weakly satisfying certain differential inequality. Although our main results in this work concern the degenerate situation when $p>2$ and the singular case $(p<2)$ will be treated in future works, several results in this section hold for $p>1$ and we will specify the range of $p$ in each statement for future reference.

Throughout this paper, the constants $C, C_{1}, \ldots$ can change line by line but they are all universal constants as they depend only on the initially fixed parameters (such as $n, m$ ). For any two quantities $A, B$, we write $A \sim B$ if there are universal positive constants $C_{1}, C_{2}$ such that $C_{1} A \leq B \leq C_{2} A$.

First of all, we recall the following Sobolev inequalities in a ball of $\mathbb{R}^{n}$ and the dependence of the constants on the size of the ball. Let $\phi$ be a function defined on $B_{1}$. By scaling, with $x=\frac{1}{R} \bar{x}$ and $\phi(x)=\bar{\phi}(\bar{x})$, we have the followings facts on the $k$ th derivatives $D^{(k)}$

$$
\left\|D_{x}^{(k)} \phi\right\|_{L^{p}\left(B_{1}\right)}^{p} \sim C R^{p k-n}\left\|D_{\bar{x}}^{(k)} \bar{\phi}\right\|_{L^{p}\left(B_{R}\right)}^{p} \text { and }\left\|D_{x} \phi\right\|_{L^{q}\left(B_{1}\right)}^{p} \sim C R^{\left(1-\frac{n}{q}\right) p}\left\|D_{\bar{x}} \bar{\phi}\right\|_{L^{q}\left(B_{R}\right)}^{p} .
$$

In particular, for $q=p_{*}=p n /(n+p)$, we easily see that $\left\|D_{x} \phi\right\|_{L^{q}\left(B_{1}\right)}^{p} \sim C R^{-n}\left\|D_{\bar{x}} \bar{\phi}\right\|_{L^{q}\left(B_{R}\right)}^{p}$. Using this in the Sobolev-Poincaré inequality on $B_{1}$, we get

$$
\|\phi\|_{L^{p}\left(B_{1}\right)}^{p} \leq C(n)\left\|D_{x} \phi\right\|_{L^{q}\left(B_{1}\right)}^{p} \Rightarrow\|\bar{\phi}\|_{L^{p}\left(B_{R}\right)}^{p} \leq C(n) R^{n+\left(1-\frac{n}{q}\right) p}\left\|D_{\bar{x}} \bar{\phi}\right\|_{L^{q}\left(B_{R}\right)}^{p}
$$

for all $p$ such that $q \leq p \leq q^{*}=q n(n-q)$.

On the other hand,

$$
\|\phi\|_{W^{-k, p^{\prime}}\left(B_{1}\right)}=\sup _{\psi} \frac{1}{\|\psi\|_{W_{0}^{k, p}\left(B_{1}\right)}} \int_{B_{1}} \phi \psi d x \leq \sup _{\psi} \frac{C}{R^{\frac{p k-n}{p}}\|\bar{\psi}\|_{W_{0}^{k, p}\left(B_{R}\right)}} \frac{1}{R^{n}} \int_{B_{R}} \bar{\phi} \bar{\psi} d \bar{x} .
$$

This also implies $\|\phi\|_{W^{-k, p^{\prime}\left(B_{1}\right)}}^{p} \sim C R^{-p k+n-p n}\|\bar{\phi}\|_{W^{-k, p^{\prime}\left(B_{R}\right)}}^{p}$.

We begin with the following lemma.

Lemma 3.1 Let $Q_{R, \rho}=B_{R} \times(-\rho, 0)$ and $u: Q_{R, \rho} \rightarrow \mathbb{R}^{m}$ be in $V_{p}\left(Q_{R, \rho}\right)$ for some $p>1$. Assume that there is a function $G \in L^{1}\left(Q_{R, \rho}\right)$ such that

$$
\left|\iint_{Q_{R, \rho}} u \phi_{t} d z\right| \leq C \iint_{Q_{R, \rho}}|G||D \phi| d z \quad \forall \phi \in C_{0}^{1}\left(Q_{R, \rho}\right) .
$$

Let $q$ be such that $p_{*}=n p /(n+p) \leq q<p$. Then, for any $\varepsilon>0$ there exist positive constants $C, C(\varepsilon)$ such that

$$
\begin{aligned}
\oiiint_{Q_{R, \rho}}\left|u-u_{R}\right|^{p} d z \leq & \varepsilon R^{p} \oiiint_{Q_{R, \rho}}|D u|^{p} d z+(C+\varepsilon) R^{p}\left(\oiiint_{Q_{R, \rho}}|D u|^{q} d z\right)^{\frac{p}{q}} \\
& +C(\varepsilon)\left(\frac{\rho}{R}\right)^{p}\left(\oiiint_{Q_{R, \rho}}|G| d z\right)^{p} .
\end{aligned}
$$


Proof: For $s, r \in(-\rho, 0)$ and $\varepsilon>0$, take $\phi=\psi(x) \eta(t)$ where $\psi \in C_{0}^{1}\left(B_{R}\right)$ and $\eta \equiv 1$ in $(s, r), \eta$ is linear in $(s-\varepsilon, s)$ and $(r, r+\varepsilon), \eta$ is zero elsewhere. By the Young inequality, the above estimate becomes

$$
\left|\int_{B_{R}}\left(\frac{1}{\varepsilon} \int_{s-\varepsilon}^{s} u d t-\frac{1}{\varepsilon} \int_{r}^{r+\varepsilon} u d t\right) \psi d x\right| \leq C\left(\iint_{Q_{R, \rho}}|G \eta| d z\right)\|\psi\|_{C_{0}^{1}\left(B_{R}\right)} .
$$

Let $l>(n+p) / p$ and $m=l-1>n / p$. By scaling, with $x=\frac{1}{R} \bar{x}$ and $\phi(x)=\bar{\phi}(\bar{x})$, we have for $\phi \in W_{0}^{m, p}\left(B_{1}\right)$ that

$$
\|\phi\|_{L^{\infty}\left(B_{1}\right)} \leq C(n)\left\|D_{x}^{(m)} \phi\right\|_{L^{p}\left(B_{1}\right)} \Rightarrow\|\bar{\phi}\|_{L^{\infty}\left(B_{R}\right)} \leq C R^{\frac{p m-n}{p}}\left\|D_{\bar{x}}^{(m)} \bar{\phi}\right\|_{L^{p}\left(B_{R}\right)},
$$

Using this for $\bar{\phi}=D \psi$, we obtain $\|\psi\|_{C_{0}^{1}\left(B_{R}\right)} \leq C R^{\frac{p l-p-n}{p}}\|\psi\|_{W_{0}^{l, p}\left(B_{R}\right)}$. Hence, letting $\varepsilon \rightarrow 0$, we obtain the following estimate for $s, r \in(-\rho, 0)$

$$
\left|\int_{B_{R}}(u(\cdot, s)-u(\cdot, r)) \psi(x) d x\right| \leq C R^{\frac{p l-p-n}{p}}\left(\iint_{Q_{R, \rho}}|G| d z\right)\|\psi\|_{W_{0}^{l, p}\left(B_{R}\right)} \quad \forall \psi \in W_{0}^{l, p}\left(B_{R}\right) .
$$

Setting $H(t, s, \cdot)=u(\cdot, s)-u(\cdot, t)$, we just proved that

$$
\|H(t, s, \cdot)\|_{W^{-l, p^{\prime}}\left(B_{R}\right)} \leq C R^{\frac{p l-p-n}{p}}\left(\iint_{Q_{R, \rho}}|G| d z\right) \quad \forall s, t \in(-\rho, 0) .
$$

Let $q$ be such that $p_{*}=n p /(n+p) \leq q<p$. We have $W^{1, q}\left(B_{1}\right) \subset L^{p}\left(B_{1}\right) \subset W^{-l, p^{\prime}}\left(B_{1}\right)$. Because $W^{1, q}\left(B_{1}\right)$ is compactly imbedded in $L^{p}\left(B_{1}\right)$, a simple argument by contradiction gives the following interpolation inequality

$$
\|\phi\|_{L^{p}\left(B_{1}\right)} \leq \varepsilon\|\phi\|_{W^{1, q}\left(B_{1}\right)}+C(\varepsilon)\|\phi\|_{W^{-l, p^{\prime}}\left(B_{1}\right)}, \quad \forall \phi \in W^{1, q}\left(B_{1}\right) .
$$

Using the norm $\|\phi\|_{W^{1, q}\left(B_{1}\right)}=\|D \phi\|_{L^{q}\left(B_{1}\right)}+\|\phi\|_{L^{p}\left(B_{1}\right)}$ and choosing $\varepsilon$ small, we have

$$
\|\phi\|_{L^{p}\left(B_{1}\right)} \leq \varepsilon\|D \phi\|_{L^{q}\left(B_{1}\right)}+C(\varepsilon)\|\phi\|_{W^{-l, p^{\prime}}\left(B_{1}\right)}, \quad \forall \phi \in W^{1, q}\left(B_{1}\right) .
$$

By a simple scaling argument, we derive from the above that

$$
R^{-n}\|\phi\|_{L^{p}\left(B_{R}\right)}^{p} \leq \varepsilon R^{\left(1-\frac{n}{q}\right) p}\|D \phi\|_{L^{q}\left(B_{R}\right)}^{p}+C(\varepsilon) R^{-l p+n-p n}\|\phi\|_{W^{-l, p^{\prime}\left(B_{R}\right)}}^{p}, \quad \forall \phi \in W^{1, q}\left(B_{R}\right) .
$$

Applying this to $H(t, s, \cdot)$ and using (3.3), we have

$$
R^{-n}\|H(t, s, \cdot)\|_{L^{p}\left(B_{R}\right)}^{p} \leq \varepsilon R^{\left(1-\frac{n}{q}\right) p}\|D H(t, s, \cdot)\|_{L^{q}\left(B_{R}\right)}^{p}+C(\varepsilon) R^{-p-p n}\left(\iint_{Q_{R, \rho}}|G| d z\right)^{p} .
$$

We now choose $s$ such that

$$
\int_{B_{R}}|D u(x, s)|^{q} d x \leq \frac{1}{\rho} \int_{-\rho}^{0} \int_{B_{R}}|D u(x, t)|^{q} d x d t
$$


Then

$$
\|D u(\cdot, s)\|_{L^{q}\left(B_{R}\right)}^{p} \leq\left(\frac{1}{\rho} \int_{-\rho}^{0} \int_{B_{R}}|D u(x, t)|^{q} d x d t\right)^{\frac{p}{q}}=\rho^{-p / q}\left(\iint_{Q_{R, \rho}}|D u|^{q} d z\right)^{\frac{p}{q}} .
$$

On the other hand, by Sobolev-Poincaré's inequality, we also have

$$
R^{-n}\left\|u(\cdot, s)-u(\cdot, s)_{R}\right\|_{L^{p}\left(B_{R}\right)}^{p} \leq C R^{\left(1-\frac{n}{q}\right) p}\|D u(\cdot, s)\|_{L^{q}\left(B_{R}\right)}^{p} \leq C R^{p}\left(\oiiint_{Q_{R, \rho}}|D u|^{q} d z\right)^{\frac{p}{q}} .
$$

Obviously,

$$
\left\|u(\cdot, t)-u(\cdot, t)_{R}\right\|_{L^{p}\left(B_{R}\right)}^{p} \leq C\|u(\cdot, t)-u(\cdot, s)\|_{L^{p}\left(B_{R}\right)}^{p}+\left\|u(\cdot, s)-u(\cdot, s)_{R}\right\|_{L^{p}\left(B_{R}\right)}^{p}
$$

and $|D H(t, s, \cdot)| \leq|D u(\cdot, t)|+|D u(\cdot, s)|$. A simple use of Hölder's inequality gives $\|D u(\cdot, t)\|_{L^{q}\left(B_{R}\right)}^{p} \leq$ $C R^{n\left(\frac{p}{q}-1\right)}\|D u(\cdot, t)\|_{L^{p}\left(B_{R}\right)}^{p}$.

Together, when such $s$ is fixed, the above yields

$$
\begin{aligned}
\frac{1}{R^{n}}\left\|u(\cdot, t)-u(\cdot, t)_{R}\right\|_{L^{p}\left(B_{R}\right)}^{p} \leq & \varepsilon R^{p-n}\|D u(\cdot, t)\|_{L^{p}\left(B_{R}\right)}^{p}+(C+\varepsilon) C R^{p}\left(\int_{Q_{R, \rho}}|D u|^{q} d z\right)^{\frac{p}{q}} \\
& +C(\varepsilon) R^{-p-p n}\left(\iint_{Q_{R, \rho}}|G| d z\right)^{p} \cdot
\end{aligned}
$$

Integrating the above over $t \in[-\rho, 0]$ and dividing by $\rho$, we get (3.2) and the proof of the lemma is then complete.

The following Poincaré type inequality is an immediate consequence of the above lemma.

Lemma 3.2 Assume as in Lemma 3.1. If $\rho=S^{2-p} R^{2}$ and $|G| \leq|D u|^{p-1}$ then for any $\varepsilon>0$ and $n p /(n+p) \leq q<p$ there exist positive constants $C, C(\varepsilon)$ such that

$$
\begin{aligned}
\oiiint_{Q_{R, \rho}}\left|u-u_{R}\right|^{p} d z \leq & \varepsilon R^{p} \oiiint_{Q_{R, \rho}}|D u|^{p} d z+(C+\varepsilon) R^{p}\left(\oiiint_{Q_{R, \rho}}|D u|^{q} d z\right)^{\frac{p}{q}} \\
& +C(\varepsilon)\left(S^{2-p} R\right)^{p}\left(\oiiint_{Q_{R, \rho}}|D u|^{p-1} d z\right)^{p} .
\end{aligned}
$$

We now consider a weak solution $u \in V_{p}\left(Q_{1,1}\right)$ to

$$
u_{t}=\operatorname{div}\left(A(u, D u) \quad \text { in } Q_{1,1}\right.
$$

The matrix $A$ is assumed to satisfy the following ellipticity conditions E) for some positive constants $\lambda, \Lambda$ and $p>1$.

By testing (3.7) with $|u-c| \phi^{2}$, with $\phi$ being a cutoff function for $Q_{\frac{1}{2} R, \frac{1}{2} \rho}, Q_{R, \rho}$, we easily get the following Caccioppoli type inequality 
Lemma 3.3 Let $u$ satisfy 3.7). For any constant vector $c \in \mathbb{R}^{m}$ and any $Q_{R, \rho} \subset Q_{1,1}$

$$
\oiiint_{Q_{\frac{1}{2} R, \frac{1}{2} \rho}}|D u|^{p} d z \leq C \frac{1}{R^{p}} \oiiint_{Q_{R, \rho}}|u-c|^{p} d z+C \frac{1}{\rho} \int_{Q_{R, \rho}}|u-c|^{2} d z .
$$

A consequence of Lemma 3.1 and the above is the following reverse Hölder inequality.

Lemma 3.4 Assume that $p>2$ and $u$ satisfies (3.7). If $\rho=S^{2-p} R^{2}$ for some $S^{p} \sim$ $\int_{Q_{R, \rho}}|D u|^{p} d z$ then we have for any given positive $\varepsilon$ and $n p /(n+p) \leq q<p$

$$
\begin{aligned}
\oiiint_{Q_{\frac{1}{2} R, \frac{1}{2} \rho}}|D u|^{p} d z \leq & \varepsilon \oiiint_{Q_{R, \rho}}|D u|^{p} d z+(C+\varepsilon)\left(\oiiint_{Q_{R, \rho}}|D u|^{q} d z\right)^{\frac{p}{q}} \\
& +C(\varepsilon)\left(\oiiint_{Q_{R, \rho}}|D u|^{p-1} d z\right)^{\frac{p}{p-1}} .
\end{aligned}
$$

Proof: For $\rho=S^{2-p} R^{2}$ we have (3.6). By Hölder inequality we estimate the last term in (3.6) as follows

$$
\left(S^{2-p} R\right)^{p}\left(\oiiint_{Q_{R, \rho}}|D u|^{p-1} d z\right)^{p} \leq R^{p}\left(\frac{\oiiint_{Q_{R, \rho}}|D u|^{p} d z}{S^{p}}\right)^{(p-2)}\left(\oiiint_{Q_{R, \rho}}|D u|^{p-1} d z\right)^{\frac{p}{p-1}} .
$$

If $p>2$ then the Young inequality $(1-(p-2) / p=2 / p)$ can apply to the last term in (3.8) to yield

$$
\frac{1}{\rho} \oiiint_{Q_{R, \rho}}\left|u-u_{R}\right|^{2} d z=S^{p-2} R^{-2} \oiiint_{Q_{R, \rho}}\left|u-u_{R}\right|^{2} d z \leq \varepsilon S^{p}+C(\varepsilon) \frac{1}{R^{p}} \oiiint_{Q_{R, \rho}}\left|u-u_{R}\right|^{p} d z .
$$

Thus, if $S^{p} \sim \int_{Q_{R}}|D u|^{p} d z$, we can combine the above estimates with (3.8) and (3.6) to obtain (3.9) if $p>2$.

The above result also holds for $2 n /(n+2)<p<2$ but we have to treat the last term in (3.8) differently.

Lemma 3.5 Assume that $2 n /(n+2)<p<2$ and $u$ satisfies (3.7). If $\rho=S^{2-p} R^{2}$ for some $S$ satisfying

$$
S^{p} \geq c(n)\left(\oiiint_{Q_{2 R, 2 \rho}}|D u|^{p} d z+\frac{1}{\rho} \oiiint_{Q_{2 R, 2 \rho}}\left|u-u_{2 R}\right|^{2} d z\right)
$$

then we have for any given positive $\varepsilon$ and $q=2 n /(n+2)$

$$
\begin{aligned}
\oiiint_{Q_{\frac{1}{2} R, \frac{1}{2} \rho}}|D u|^{p} d z \leq & \varepsilon \oiiint_{Q_{R, \rho}}|D u|^{p} d z+(C+\varepsilon)\left(\oiiint_{Q_{2 R, 2 \rho}}|D u|^{q} d z\right)^{\frac{p}{q}} \\
& +C(\varepsilon) S^{p} .
\end{aligned}
$$


Proof: Let $\chi(x)$ be a smooth function in $x$ with compact support in $B_{R}\left(x_{0}\right)$ such that $|D \chi| \leq c / R$. We define

$$
u_{x_{0}, R}^{\chi}(t)=\int_{B_{R}\left(x_{0}\right)} u(x, t) d x / \int_{B_{R}\left(x_{0}\right)} \chi(x) d x .
$$

For any $s, t$ such that $-\rho<s<t<0$, we test (3.7) with $\left(u_{x_{0}, R}^{\chi}(t)-u_{x_{0}, R}^{\chi}(s)\right) \chi(x) \eta(\tau), \eta$ is defined as in Lemma 3.1, to get the following.

$$
\left|u_{x_{0}, R}^{\chi}(t)-u_{x_{0}, R}^{\chi}(s)\right|^{2} \leq C \frac{t-s}{R^{n+2}} \int_{Q_{R, \rho}}|G|^{2} d z \leq C \frac{t-s}{R^{n+2}} \int_{Q_{R, \rho}}|D u|^{2 p-2} d z .
$$

Thus, if $|t-s| \leq \rho=S^{2-p} R^{2}$ then

$$
\frac{1}{\rho}\left|u_{x_{0}, R}^{\chi}(t)-u_{x_{0}, R}^{\chi}(s)\right|^{2} \leq C S^{2-p} \oiiint_{Q_{R, \rho}}|D u|^{2 p-2} d z .
$$

Obviously, for any $s \in(-\rho, 0)$

$$
\begin{aligned}
\frac{1}{\rho} \int_{Q_{R, \rho}}\left|u-u_{R}\right|^{2} d z & \leq \frac{1}{\rho} \int_{Q_{R}}\left|u-u_{x_{0}, R}^{\chi}(s)\right|^{2} d z \\
& \leq \frac{1}{\rho} \int_{Q_{R}}\left|u-u_{x_{0}, R}^{\chi}(t)\right|^{2} d z+\frac{1}{\rho} \sup _{t \in(-\rho, 0)}\left|u_{x_{0}, R}^{\chi}(t)-u_{x_{0}, R}^{\chi}(s)\right|^{2} .
\end{aligned}
$$

The last term can be estimated as in (3.12) while the first term on the right is bounded by $\iint_{Q_{R, \rho}}\left|u-u_{x_{0}, R}^{\chi}(t)\right|^{2} d z \leq\left(\sup _{t} \int_{B_{R}}\left|u-u_{x_{0}, R}^{\chi}(t)\right|^{2} d x\right)^{1-\frac{q}{2}} \int_{\rho}^{0}\left(\int_{B_{R}}\left|u-u_{x_{0}, R}^{\chi}(t)\right|^{2} d x\right)^{\frac{q}{2}} d t$ where $q=2 n /(n+2)$. The last factor can be bounded via Poincaré-Sobolev's inequality (in the $x$ variable) by

$$
\int_{\rho}^{0}\left(\int_{B_{R}}\left|u-u_{x_{0}, R}^{\chi}(t)\right|^{2} d x\right)^{\frac{q}{2}} d t \leq \iint_{Q_{R, \rho}}|D u|^{q} d z .
$$

From the equation (3.7) for $u$, we easily obtain

$$
\begin{aligned}
\sup _{t \in(-\rho, 0)} \int_{B_{R}} \mid u & -\left.u_{x_{0}, R}^{\chi}(t)\right|^{2} d x \leq \sup _{t \in(-\rho, 0)} \int_{B_{R}}\left|u-u_{2 R}\right|^{2} d x \\
& \leq \frac{1}{\rho} \iint_{Q_{2 R, 2 \rho}}\left|u-u_{2 R}\right|^{2} d z+\frac{1}{R^{p}} \iint_{Q_{2 R, 2 \rho}}\left|u-u_{2 R}\right|^{p} d z .
\end{aligned}
$$
have

Applying Hölder inequality to the right hand side of the Poincaré inequality (3.6), we

$$
\left.\frac{1}{R^{p}} \oiiint_{Q_{2 R, 2 \rho}}\left|u-u_{2 R}\right|^{p} d z \leq C \int_{Q_{2 R, 2 \rho}}|D u|^{p} d z+C S^{(2-p) p}\left(\oiiint_{Q_{2 R, 2 \rho}}|D u|^{p} d z\right)\right)^{p-1} .
$$


By (3.10), the right hand side is bounded by $C S^{p}$. Thus,

$$
\frac{1}{R^{p}} \iint_{Q_{2 R, 2 \rho}}\left|u-u_{2 R}\right|^{p} d z=\frac{S^{2-p} R^{n+2}}{R^{p}} \oiiint_{Q_{2 R, 2 \rho}}\left|u-u_{2 R}\right|^{p} d z \leq C R^{n+2} S^{2} .
$$

(3.10) also gives

$$
\frac{1}{\rho} \iint_{Q_{2 R, 2 \rho}}\left|u-u_{2 R}\right|^{2} d z=\frac{\left|Q_{2 R, 2 \rho}\right|}{\rho} \int_{Q_{2 R, 2 \rho}}\left|u-u_{2 R}\right|^{2} d z \leq C\left|Q_{R, \rho}\right| S^{p}=C R^{n+2} S^{2} .
$$

The above estimates and (3.13) yield

$$
\left(\sup _{t \in(-\rho, 0)} \int_{B_{R}}\left|u-u_{x_{0}, R}^{\chi}(t)\right|^{2} d x\right)^{1-q / 2} \leq C\left(R^{n+2} S^{2}\right)^{\frac{2}{n+2}}=C R^{2} S^{\frac{4}{n+2}} .
$$

Hence,

$$
\begin{aligned}
\frac{1}{\rho} \oiiint_{Q_{R, \rho}}\left|u-u_{R}\right|^{2} d z & \leq C S^{p-2+\frac{4}{n+2}} \oiiint_{Q_{R, \rho}}|D u|^{q} d z=C S^{p-q} \oiiint_{Q_{R, \rho}}|D u|^{q} d z \\
& \leq \varepsilon S^{p}+C(\varepsilon)\left(\oiiint_{Q_{R, \rho}}|D u|^{q} d z\right)^{p / q} .
\end{aligned}
$$

From the assumption on $S$ and the Caccioppoli inequality (3.8) we derive the desired reverse Hölder inequality in this case.

Finally, in order to obtain certain uniform continuity for the integrals of gradients of weak solutions, we will need the following measure theoretic result which could be of interest in its own right.

Lemma 3.6 Let $F, G_{k}(k=1, \ldots, M)$ be integrable functions defined on $Q_{1,1}$ and $\alpha, \beta, m_{k}$ be real numbers with $\alpha+1, \beta>0$ and $m_{k} \in(0,1)$. Assume that for any scaled cylinders $Q_{R, \rho} \subset Q_{2 R, 2 \rho} \subset Q_{1,1}$ with $\rho=S^{\alpha} R^{\beta}$ and $S \sim \int_{Q_{R, \rho}} F d z$ the following holds.

$$
\oiiint_{Q_{R, \rho}} F d z \leq \varepsilon \oiiint_{Q_{2 R, 2 \rho}} F d z+\sum_{k=1}^{M}\left(\oiiint_{Q_{2 R, 2 \rho}} G_{k} d z\right)^{1 / m_{k}} .
$$

If $\varepsilon>0$ is sufficiently small (depending on $\|F\|_{L^{1}\left(Q_{1,1}\right)}, \alpha, \beta, m_{k}$ ) then for any subset $A$ of $Q_{\frac{1}{2}, \frac{1}{2}}, m \in(0,1)$ and

$$
t^{\alpha+1} \geq \oiiint_{Q_{1,1}} F d z
$$

there is a positive constant $C=C(n)$ such that

$$
\iint_{\Phi_{t}} F d z \leq C t^{1-m} \iint_{\Phi_{t}} F^{m} d z+C \sum_{k=1}^{M} t^{1-m_{k}} \iint_{\Gamma_{t}^{k}} G_{k} d z .
$$

Here, for any $t>0$, we set $\Phi_{t}=\{z: z \in A$ and $F(z)>t\}$ and $\Gamma_{t}^{k}=\{z: z \in$ $A$ and $\left.G_{k}(z)>t^{m_{k}}\right\}$. 
Proof: For simplicity, we will consider the case when $M=1$ since it is easy to extend the argument to the case $M>1$.

Let $P=Q_{\frac{1}{2}, \frac{1}{2}}$ and $t=\lambda_{0} S$ with $\lambda_{0}=\lambda_{0}(n)$ being a constant to be determined later. We have from (3.15)

$$
\lambda_{0}^{\alpha+1} S^{\alpha+1} \geq \oiiint_{Q_{1,1}} F d z
$$

Define

$$
J(r)=\oiiint_{Q_{r, S^{\alpha} r^{\beta}}} F d z, \quad r>0 .
$$

Obviously, for $r \in\left[\frac{1}{10}, \frac{1}{2}\right]$ and $\lambda_{0}=\lambda_{0}(n)$ sufficiently small we can have

$$
J(r) \leq S^{-\alpha} C(n) \oiiint_{Q_{1,1}} F d z=S C(n) \frac{1}{S^{\alpha+1}} \oiiint_{Q_{1,1}} F d z \leq \frac{1}{4} S \quad \forall r \in\left[\frac{1}{10}, \frac{1}{2}\right] .
$$

Consider a point $z \in P$ such that $F(z)>S$. Lebesgue's theorem yields $\lim _{r \rightarrow 0} J(r)>S$. Thus, by the continuity of the integral and the above inequality, we can find $r(z) \in\left(0, \frac{1}{10}\right)$ such that $J(r(z))=\frac{1}{2} S$ and $J(\rho) \leq \frac{1}{2} S$ for any $\rho \in\left[r(z), \frac{1}{10}\right]$. This and (3.18) imply that $J(2 r(z))$ and $J(5 r(z))$ are bounded by $\frac{1}{2} S$. Moreover, there is a constant $c_{0}$ depending on $n$ such that $J(r(z)) \leq c_{0} J(2 r(z))$ and therefore $J(2 r(z)) \geq \frac{1}{2} c_{0}^{-1} S$.

Hence, for $\rho(z)=S^{\alpha} r^{\beta}(z)$

$$
S \sim \oiiint_{Q_{2 r(z), S^{\alpha}(2 r)^{\beta}(z)}} F d z \text { and } \oiiint_{Q_{5 r(z), S^{\alpha}(5 r)^{\beta}(z)}} F d z \leq \frac{1}{2} S .
$$

We apply the Calderon-Zygmund lemma to $P$ to obtain a countable family of disjoint subcubes $\left\{Q_{i}\right\}=\left\{Q_{2 r\left(z_{i}\right), S^{\alpha}(2 r)^{\beta}\left(z_{i}\right)}\right\}$ such that $\{z: z \in P$ and $F(z)>S\} \subset \cup_{i} \hat{Q}_{i}$. Here, $\hat{Q}_{i}=Q_{5 r\left(z_{i}\right), S^{\alpha}(5 r)^{\beta}\left(z_{i}\right)}$.

Let $A$ be a subset of $P$ and $\Phi_{t}=\{z: z \in A$ and $F(z)>t\}$ and $\Gamma_{t}=\{z: z \in$ $A$ and $\left.G(z)>t^{m_{1}}\right\}$. We will only consider subcubes $Q_{i}$ 's such that $Q_{i} \cap A \neq \emptyset$.

For such a subcube, $Q_{2 r\left(z_{i}\right), S^{\alpha}(2 r)^{\beta}\left(z_{i}\right)} \subset Q_{4 r\left(z_{i}\right), S^{\alpha}(4 r)^{\beta}\left(z_{i}\right)} \subset Q_{1,1}$ and by (3.19) we see that (3.14) holds and we have two cases (with $\bar{Q}_{r, \rho}=Q_{2 r, 2 \rho}$ )

$$
\oiiint_{Q_{i}} F d z \leq 2 \varepsilon \oiint_{\bar{Q}_{i}} F d z \text { or } \oiint_{Q_{i}} F d z \leq 2\left(\oiiint_{\bar{Q}_{i}} G d z\right)^{1 / m_{1}} .
$$

If the second case of (3.20) holds then because $S \sim \int_{Q_{i}} F d z$ we have the following

$$
c_{1}(n)^{-m_{1}} S^{m_{1}}<\left(\oiiint_{Q_{i}} F d z\right)^{m_{1}} \leq 2^{m_{1}} \oiiint_{\bar{Q}_{i}} G d z \leq c_{2}(n) 2^{m_{1}} \oiiint_{\hat{Q}_{i}} G d z
$$

Hence, by splitting the integral on $\hat{Q}_{i}$ into those on $\hat{Q}_{i} \cap \Gamma_{t}$ and $\hat{Q}_{i} \backslash \Gamma_{t}$, we have for some $c=c(n)$

$$
S^{m_{1}}\left|\hat{Q}_{i}\right|<(2 c)^{m_{1}} \iint_{\hat{Q}_{i} \cap \Gamma_{t}} G d z+(2 c)^{m_{1}} \iint_{\hat{Q}_{i} \backslash \Gamma_{t}} G d z .
$$


This gives

$$
S^{m_{1}}\left|\hat{Q}_{i}\right|<(2 c)^{m_{1}} \iint_{\hat{Q}_{i} \cap \Gamma_{t}} G d z+(2 c)^{m_{1}} t^{m_{1}}\left|\hat{Q}_{i}\right|
$$

and furthermore

$$
S^{m_{1}}\left|\hat{Q}_{i}\right| \leq c_{3}(n) \iint_{\hat{Q}_{i} \cap \Gamma_{t}} G d z
$$

if $\lambda_{0}=t / S$ is sufficiently small (such that $(2 c)^{m_{1}} t^{m_{1}} \leq \frac{1}{2} S^{m_{1}}$ and (3.18) still holds). We then fix such $\lambda_{0}$.

Arguing similarly, with $G, m_{1}$ now being $\varepsilon F$ and 1 , we see that if the first case of (3.20) holds and $\varepsilon$ is small then

$$
S\left|\hat{Q}_{i}\right| \leq 4 \varepsilon \iint_{\hat{Q}_{i} \cap \Phi_{S}} F d z .
$$

Together, we have

$$
S\left|\hat{Q}_{i}\right| \leq 4 \varepsilon \iint_{\hat{Q}_{i} \cap \Phi_{S}} F d z+c_{3}(n) S^{1-m_{1}} \iint_{\hat{Q}_{i} \cap \Gamma_{t}} G d z .
$$

Since $\Phi_{S} \subset \cup_{i} \hat{Q}_{i}$, we now have by (3.19) and the fact that $Q_{i}$ 's are disjoint

$$
\iint_{\Phi_{S}} F d z \leq \sum_{\hat{Q}_{i} \cap \Phi_{S} \neq \emptyset} \iint_{\hat{Q}_{i}} F d z \leq \frac{1}{2} S \sum_{\hat{Q}_{i} \cap \Phi_{S} \neq \emptyset}\left|\hat{Q}_{i}\right|=C(n) S \sum_{i}\left|Q_{i}\right|=C(n) S\left|\cup Q_{i}\right| .
$$

By Vitali's covering lemma, we can find a subsequence of disjoint subcubes $\left\{\Pi_{i}\right\}$ of $\left\{\hat{Q}_{i}\right\}$ such that $\cup Q_{i} \subset \cup \hat{\Pi}_{i}$ and therefore

$$
\left|\cup Q_{i}\right| \leq\left|\cup \hat{\Pi}_{i}\right| \leq \sum\left|\hat{\Pi}_{i}\right| \leq C_{1}(n) \sum\left|\Pi_{i}\right| .
$$

Thus, as $\Pi$ 's are disjoint, we have from the above estimates for $\Pi_{i}=\hat{Q}_{i}$ that

$$
\begin{aligned}
\iint_{\Phi_{S}} F d z & \leq C_{2}(n) S \sum\left|\Pi_{i}\right| \leq C_{3}(n) \sum\left(4 \varepsilon \iint_{\Pi_{i} \cap \Phi_{S}} F d z+S^{1-m_{1}} \iint_{\Pi_{i} \cap \Gamma_{t}} G d z\right) \\
& \leq C_{3}(n) 4 \varepsilon \iint_{\Phi_{S}} F d z+C_{3}(n) S^{1-m_{1}} \iint_{\Gamma_{t}} G d z .
\end{aligned}
$$

On the other hand, as $\lambda_{0}$ is small, $t<S$ and therefore $\Phi_{S} \subset \Phi_{t}$. We then have

$$
\iint_{\Phi_{t} \backslash \Phi_{S}} F d z \leq S^{1-m} \iint_{\Phi_{t}} F^{m} d z \leq 2 C_{4}(n) t^{1-m} \iint_{\Phi_{t}} F^{m} d z .
$$

Hence, by choosing $\varepsilon$ sufficiently small, we get

$$
\iint_{\Phi_{t}} F d z \leq C t^{1-m} \iint_{\Phi_{t}} F^{m} d z+C t^{1-m_{1}} \iint_{\Gamma_{t}} G d z
$$

This completes the proof.

Remark 3.7 Note that the number $S$ in the proof is fixed and needs only satisfy (3.16). 
We then have the following result on the uniform continuity of integrals.

Lemma 3.8 Let $\left\{F^{(i)}\right\}$ and $\left\{G_{k}^{(i)}\right\}, k=1, \ldots, M$, be bounded sequences of functions in $L^{1}\left(Q_{1,1}\right)$ satisfying (3.14) of Lemma 3.6. Assume that the sequences $\left\{G_{k}^{(i)}\right\}$ are weakly convergent in $L^{1}\left(Q_{1,1}\right)$. If $\varepsilon$ is sufficiently small then the integrals of $F^{(i)}$ are uniformly continuous in the following sense: for any given $\delta>0$ there is $\mu(\delta)>0$ such that

$$
\iint_{A} F^{(i)} d z<\delta \text { for all } i \text { if } A \subset Q_{\frac{1}{2}, \frac{1}{2}} \text { and }|A| \leq \mu(\delta) .
$$

Proof: We can again consider the case $M=1$. Fix a $t$ satisfying (3.15) of Lemma 3.6. We have shown that if $\varepsilon$ is small enough then we have for $F=F^{(i)}$ and $G=G^{(i)}$.

$$
\begin{aligned}
\iint_{A} F d z & =\iint_{A \backslash \Phi_{t}} F d z+\iint_{\Phi_{t}} F d z \leq t|A|+C t^{1-m} \iint_{\Phi_{t}} F^{m} d z+C t^{1-m_{1}} \iint_{\Gamma_{t}} G d z \\
& \leq t|A|+C t^{1-m} \iint_{A} F^{m} d z+C t^{1-m_{1}} \iint_{A} G d z .
\end{aligned}
$$

Since $\left|F^{(i)}\right|^{m}, G^{(i)}$ are weakly convergent in $L^{1}\left(Q_{1,1}\right)$, we can apply [2, Corollary IV.11] on the uniform continuity of integrals to see that the last two integrals are uniformly small if $|A|$ is small. The assertion then follows easily.

Another consequence of Lemma 3.6 is the following higher integrability result.

Lemma 3.9 Let $\left\{F^{(i)}\right\}$ and $\left\{G_{k}^{(i)}\right\}, k=1, \ldots, M$, be bounded sequences of functions in $L^{1}\left(Q_{1,1}\right)$ satisfying 3.14) of Lemma 3.6 (with $\left.\rho=S^{\alpha} R^{\beta}\right)$. If $\varepsilon$ is sufficiently small then there is some $r>1$ such that if $G_{i} \in L^{r-m_{i}+1}\left(Q_{1,1}\right)$ then the following estimate holds

$$
\iint_{Q_{\frac{1}{2}, \frac{1}{2}}} F^{r} d z \leq C\left(\iint_{Q_{1,1}} F d z\right)^{\frac{r-1}{1+\alpha}+1}+C \Sigma \iint_{Q_{1,1}} G_{i}^{r-m_{i}+1} d z .
$$

Proof: For $m \in(0,1)$ and $r>1$, we define

$$
\phi(t)=\iint_{\Phi_{t}} F^{m} d z, \quad \omega_{i}(t)=\iint_{\Gamma_{t}} G_{i} d z
$$

and

$$
I_{r}(t)=\iint_{\Phi_{t}} F^{r} d z
$$

For $A=Q_{\frac{1}{2}, \frac{1}{2}}$, the assertion in Lemma 3.6 can be written as

$$
-\int_{t}^{\infty} \tau^{1-m} d \phi(\tau) \leq C\left[t^{1-m} \phi(t)+\Sigma_{i} t^{1-m_{i}} \omega_{i}(t)\right] \quad \forall t \geq a:=\left(\oiiint_{Q_{1,1}} F d z\right)^{\frac{1}{\alpha+1}} .
$$

A simple modification of the Gehring lemma in [4, Lemma 6.3, p.200] provides some $r>1$ such that

$$
-\int_{a}^{\infty} u^{r-m} d \phi(u) \leq-C a^{r-1} \int_{a}^{\infty} u^{1-m} d \phi(u)-C \Sigma \int_{a}^{\infty} u^{r-m_{i}} d \omega_{i}(u) .
$$


This gives (see [4])

$$
\iint_{Q_{\frac{1}{2}, \frac{1}{2}}} F^{r} d z \leq C a^{r-1} \iint_{Q_{1,1}} F d z+C \Sigma \iint_{Q_{1,1}} G_{i}^{r-m_{i}+1} d z .
$$

The definition of $a$ then gives the desired (3.21).

We now consider a sequence of vector functions $u_{k}$ which almost solve (3.7) in the following sense:

$$
\left|\iint_{Q_{R, \rho}}-u \phi_{t}+\left\langle A_{k}(u, D u), D \phi\right\rangle d z\right| \leq \delta\|D u\|_{L^{p}\left(Q_{R, \rho}\right)}^{\frac{p}{q}}\|D \phi\|_{L^{p}\left(Q_{R, \rho}\right)}
$$

for all $\phi \in V_{p}^{0}\left(Q_{R, \rho}\right), Q_{R, \rho} \subset Q_{1,1}$.

Lemma 3.10 Assume that $2 n /(n+2)<p<2$. Let $\left\{u_{k}\right\}$ be a sequence of vector functions satisfying (3.22) and the norms $\left\|D u_{k}\right\|_{L^{p}\left(Q_{1,1}\right)}$ are uniformly bounded. The sequence of matrices $A_{k}$ is assumed to satisfied the ellipticity condition E) of Section Q If $\delta$ is sufficiently small then the integrals of $\left|D u_{k}\right|^{p}$ are uniformly continuous.

Proof: We will apply Lemma 3.8 here by taking $F^{(i)}=\left|D u_{i}\right|^{p}$ and $G_{k}^{(i)}(k=1,2)$ to be either $\left|D u_{i}\right|^{q}$ or $\left|D u_{i}\right|^{p-1}$ with $q=n p /(n+p)$. Consider first the case $p>2$. It is easy to see that $u_{i}$ satisfies the Poincaré and Caccioppoli type inequalities of Lemma 3.1 and Lemma 3.3. Hence the reverse Hölder inequality (3.9) of Lemma 3.4 holds for $D u_{i}$ with $\varepsilon=2 \delta$ and

$$
\rho=S^{2-p} R^{2} \text { and } S^{p} \sim \oiiint_{Q_{R, \rho}}|D u|^{p} d z .
$$

Hence, the assumption (3.14) of Lemma 3.6 is verified with $\alpha, S$ there being $(2-p) / p, S^{p}$ respectively, $\rho=S^{\alpha} R^{\beta}$ and $\beta=2$.

As the norms $\left\|D u_{k}\right\|_{L^{p}\left(Q_{1,1}\right)}$ are uniformly bounded, $G_{i}$ 's are uniformly bounded in $L^{r}$ for some $r>1$ and they are weakly convergent in $L^{1}$. Lemma 3.8 then applies here to give our lemma if $\varepsilon$, or $\delta$, is sufficiently small.

If $p<2$, as in the proof of Lemma 3.6, we fix a number $S$ such that (see (3.16))

$$
\lambda_{0}^{\frac{2}{p}} S^{2} \geq\left(\oiiint_{Q_{1,1}}\left|D u_{k}\right|^{p} d z+\frac{1}{S^{2-p}} \oiiint_{Q_{1,1}}\left|u_{k}-\left(u_{k}\right)_{Q_{1,1}}\right|^{2} d z\right)
$$

Define (see (3.17))

$$
J(r)=\oiiint_{Q_{r, S^{2-p} r^{2}}}\left|D u_{k}\right|^{p} d z+\frac{1}{S^{2-p} r^{2}} \oiiint_{Q_{r, S^{2-p_{r}}}}\left|u_{k}-\left(u_{k}\right)_{Q_{1,1}}\right|^{2} d z .
$$

We will be interested in the set where $\left|D u_{k}\right|^{p}>S^{p}$. At each point $z$ of this set, the argument leading to (3.19) in the proof allows us to find a cylinder $Q_{R, S^{2-p} R^{2}}(z)$ and a positive constant $c_{1}(n)$ such that

$$
c_{1}(n)\left(\oiiint_{Q_{R, S^{2}-R^{2}}}|D u|^{p} d z+\frac{1}{S^{2-p} R^{2}} \oiiint_{Q_{R, S^{2}-p_{R}}}\left|u-u_{R}\right|^{2} d z\right) \leq S^{p} .
$$


Therefore, the condition (3.10) on $S$ is verified and a reverse Hölder inequality (3.14) for $F^{(k)}=\left|D u_{k}\right|^{p}$ is available again. Noting that $S$ is fixed, we see that the proof can continue as before.

We also have the following $L^{q}$ estimates for $D u$ as a result of Lemma 3.9.

Lemma 3.11 Assume that $p>2 n /(n+2)$. Consider a vector functions $u$ satisfying (3.22) with $A_{k}$ is assumed to satisfied the ellipticity condition E) of Section Q. If $\delta$ is sufficiently small then there is $\varepsilon>0$ such that

$$
R^{p+\varepsilon} \oiiint_{Q_{\frac{1}{2} R, \frac{1}{2} R^{p}}}|D u|^{p+\varepsilon} d z \leq C
$$

for some constant $C$ depending on $R^{p} \int_{Q_{R, R^{p}}}|D u|^{p} d z$. If $2 n /(n+2)<p<2$, the above constant $C$ also depends on $\frac{1}{R^{p}} \oiiint_{Q_{R, R^{p}}}\left|u-u_{R}\right|^{2} d z$.

Proof: We make the scaling $x \rightarrow x / R, t \rightarrow t / R^{p}$ in the equation for $u$ and need only show that $(3.23)$ when $R=1$. We now set $F=|D u|^{p}$ and $G_{i}$ be either $|D u|^{p-1}$ or $|D u|^{q}$ with $q=n p /(n+p)$. Accordingly, $m_{i}$ will be either $(p-1) / p$ or $q / p=n /(n+p)$ respectively. Thus, $G_{i}=F^{m_{i}}$ and belongs to $L^{r-m_{i}+1}$ if $\left(r-m_{i}+1\right) m_{i} \leq 1$. We can find such $r>1$ if $\left(2-m_{i}\right) m_{i}<1$ but this requirement is just one of the followings

$$
\left(2-\frac{p-1}{p}\right) \frac{p-1}{p}<1 \Leftrightarrow p^{2}-1<p^{2} \text { and }\left(2-\frac{n}{n+p}\right) \frac{n}{n+p}<1 \Leftrightarrow n(n+2 p)<(n+p)^{2} .
$$

Thus, Lemma 3.9 applies here with $\alpha=(2-p) / p$ and $r=1+\varepsilon / p$ to give (3.23) when $R=1$. The dependence of the constant $C$ on $\frac{1}{R^{p}} \int_{Q_{R, R^{p}}}\left|u-u_{R}\right|^{2} d z$ comes from the choice of $S$ in the proof of Lemma 3.10 .

Remark 3.12 When $p>2$, it is easy to see that the quantity

$$
\left(R^{p+\varepsilon} \int_{Q_{\frac{1}{2} R, \frac{1}{2} R^{p}}}|D u|^{p+\varepsilon} d z\right)^{\frac{p}{p+\varepsilon}}
$$

can be bounded by

$$
C_{0} \max \left\{\left(R^{p} \oiiint_{Q_{R, R^{p}}}|D u|^{p} d z\right)^{\frac{p(2+\varepsilon)}{2(p+\varepsilon)}}, R^{p} \oiiint_{Q_{R, R^{p}}}|D u|^{p} d z\right\}
$$

for some constant $C_{0}$ independent of $D u$. We also remark that the exponent $\frac{p(2+\varepsilon)}{2(p+\varepsilon)}>1$ when $p>2$. 


\section{The approximation lemmas}

In this section, we establish one of the main tools of our work - the nonlinear approximation lemma for $p$-Laplacian systems. To begin, let us fix a cylinder $Q_{R}=B_{R} \times\left[-R^{p}, 0\right]$ and consider two collections $\mathcal{A}$ of matrix-valued functions $A$ and $\mathcal{B}$ of vector valued functions $B$ satisfying the followings

a.1) There are positive constants $\lambda, \Lambda$ such that the ellipticity condition (2.2) in E) holds for each $A \in \mathcal{A}$.

a.2) For any $B \in \mathcal{B}, B(u, \zeta)$ is Lipschitz in $u$ and there is a positive constant $C$ such that

$$
|B(u, \zeta)| \leq C+C|\zeta|^{p-1} .
$$

a.3) For each $A \in \mathcal{A}, B \in \mathcal{B}$ and any given function $g \in C^{1}\left(Q_{\rho}\right)$, where $Q_{\rho} \subset Q_{R}$, the system

$$
\iint_{Q_{\rho}}-u \phi_{t}+\langle A(u, D u), D \phi\rangle-\langle B(u, D u), \phi\rangle d z=0, \forall \phi \in C_{0}^{1}\left(Q_{\rho}\right), \quad u=g \text { on } S_{\rho}
$$

has a bounded weak solution $u$ with $\|u\|_{L^{\infty}\left(Q_{\rho}\right)} \leq C\left(\|g\|_{L^{\infty}\left(Q_{\rho}\right)}\right)$.

a.4) (Monotonicity) There is a positive constant $\lambda_{0}$ such that

$$
\left.\iint_{Q_{\rho}}\langle A(u, D u)-A(v, D v), D u-D v\rangle d z \geq \lambda_{0} \iint_{Q_{\rho}} \mid D u-D v\right)\left.\right|^{p} d z
$$

for any $u, v \in V_{p}\left(Q_{\rho}\right)$.

The monotonicity condition a.4) has been frequently assumed in literature concerning the uniqueness of weak solutions to the systems described in a.3). We will state the first version of our nonlinear approximation results under this condition in order to streamline our presentation and ideas. Later, we will replace a.4) by more practical assumptions and the proof will be similar modulo some technical modifications.

We will first prove that

Proposition 4.1 Assume a.1)- a.4) and $p>2 n /(n+2)$. For any given $M, \varepsilon>0$ and $\beta>1$ there exists $\delta \in(0,1]$ that depends only on $\lambda, \Lambda, M, \varepsilon$ such that if $A \in \mathcal{A}, B \in \mathcal{B}$ and $u \in V_{p}\left(Q_{R}\right)$ satisfying

$$
\oiiint_{Q_{R}}|u|^{2} d z+R^{p} \oiiint_{Q_{R}}|D u|^{p} d z \leq M
$$

and

$$
\left|\iint_{Q_{R}}-u \phi_{t}+\langle A(u, D u), D \phi\rangle-\langle B(u, D u), \phi\rangle d z\right| \leq \delta\|D u\|_{L^{p}\left(Q_{R}\right)}^{\frac{p}{q}}\|D \phi\|_{L^{p}\left(Q_{R}\right)}
$$

for all $\phi \in V_{p}^{0}\left(Q_{R}\right)$, then either

$$
R^{p} \oiiint_{Q_{R}}|D u|^{p} d z<\varepsilon
$$


or there exists $v \in V_{p}\left(Q_{R / 2}\right)$ such that

$$
\oiiint_{Q_{R / 2}}|v|^{2} d z+R^{p} \oiiint_{Q_{R / 2}}|D v|^{p} d z \leq C\left(\lambda, \Lambda, \lambda_{0}, M\right)
$$

and

$$
\iint_{Q_{R / 2}}-v \phi_{t}+\langle A(v, D v), D \phi\rangle-\langle B(v, D v), \phi\rangle d z=0
$$

for all $\phi \in C_{0}^{1}\left(Q_{R / 2}\right)$, and

$$
\left\{\begin{array}{l}
\oiiint_{Q_{R / 2}}|v-u|^{2} d z+\oiiint_{Q_{R / 2}}|v-u|^{p} d z \leq \varepsilon R^{p} \oiiint_{Q_{R}}|D u|^{p} d z, \\
\oiiint_{Q_{R / 2}}|D v|^{p} d z \leq \beta \int_{Q_{R / 2}}|D u|^{p} d z .
\end{array}\right.
$$

Proof: For simplicity we will present the proof when $B$ is identically zero. It is not difficult to see that the presence of $B$, satisfying our assumptions, will introduce some extra terms which can be easily treated by the same argument and a simple use of Young's inequality.

The proof is by contradiction, we then assume that there exist $\varepsilon_{0}>0$ and sequences $\left\{A_{k}\right\},\left\{u_{k}\right\},\left\{\varepsilon_{k}\right\}$ and cylinders $Q_{R_{k}}\left(x_{k}, t_{k}\right)$ such that for $Q_{R_{k}}=Q_{R_{k}, R_{k}^{p}}\left(x_{k}, t_{k}\right)$ we have

$$
\left|\iint_{Q_{R_{k}}}-u_{k} \phi_{t}+\left\langle A_{k}\left(u_{k}, D u_{k}\right), D \phi\right\rangle d z\right| \leq \varepsilon_{k}\left\|D u_{k}\right\|_{L^{p}\left(Q_{R_{k}}\right)}^{\frac{p}{q}}\|D \phi\|_{L^{q}\left(Q_{R_{k}}\right)}
$$

for all $\phi \in V_{p}^{0}\left(Q_{R_{k}}\right)$ but

$$
\limsup _{k \rightarrow \infty} R_{k}^{p} \bigoplus_{Q_{R_{k}}}\left|D u_{k}\right|^{p} d z>0
$$

and

$$
\oiiint_{Q_{R_{k} / 2}}\left|v-u_{k}\right|^{2} d z+\oiiint_{Q_{R_{k} / 2}}\left|v-u_{k}\right|^{p} d z>\varepsilon_{0} R_{k}^{p} \oiiint_{Q_{R_{k}}}\left|D u_{k}\right|^{p} d z
$$

for all $v$ satisfying

$$
\iint_{Q_{R_{k}}}-v \phi_{t}+\left\langle A_{k}(v, D v), D \phi\right\rangle d z=0 \quad \text { for all } \phi \in V_{p}^{0}\left(Q_{R_{k} / 2}\right) .
$$

We then make a change of variables

$$
\widetilde{u}_{k}(x, t)=u_{k}\left(x_{k}+R_{k} x, t_{k}+R_{k}^{p} t\right), \quad(x, t) \in Q_{1} .
$$

By the boundedness assumption (4.2), the norms $\left\|u_{k}\right\|_{V_{p}\left(Q_{R_{k}}\right)}$ are uniformly bounded by $M$ and so are $\left\|\widetilde{u}_{k}\right\|_{V_{p}\left(Q_{1}\right)}$. Thus, by scaling and translation, we can assume $R=1$ in (4.7)-(4.9) and note that (4.2) and (4.3) remain. This also proves that $\delta$ is independent of $R$.

For any positive real number $h$ and any vector valued function $f$ in $L^{1}\left(Q_{1}\right)$, we denote by $f_{h}=J_{h} * f$ the standard mollifier of $f$. That is, for some smooth nonnegative function $J$ with compact support in the unit ball $Q_{1}$ of $\mathbb{R}^{n+1}$ and $\|J\|_{L^{1}\left(\mathbb{R}^{n+1}\right)}=1$, we write

$$
f_{h}(Z)=J_{h} * f(Z)=\frac{1}{h^{n+1}} \iint_{Q_{1}} J\left(\frac{|Z-z|}{h}\right) f(z) d z, \quad Z \in \mathbb{R}^{n+1} .
$$


Let $\left\{h_{k}\right\}$ be some sequence of positive reals converges to 0 and $g_{k}=\left(u_{k}\right)_{h_{k}}$, which is in $C^{1}\left(Q_{1}\right)$. We then define $U_{k}$ to be the solutions of

$$
\begin{cases}\iint_{Q_{\frac{2}{3}}}-U_{k} \phi_{t}+\left\langle A_{k}\left(U_{k}, D U_{k}\right), D \phi\right\rangle d z=0 & \forall \phi \in C_{0}^{1}\left(Q_{\frac{2}{3}}\right) \\ U_{k}=g_{k} & \text { on } S_{\frac{2}{3}} .\end{cases}
$$

Note that, by a.3), $\left\|U_{k}\right\|_{\infty} \leq C\left(\left\|g_{k}\right\|_{\infty}\right)$.

The following claims provide a contradiction to (4.8) and (4.9) and prove our proposition.

Claim I: There is a constant $C$ such that $\left\|D U_{k}\right\|_{L^{p}\left(Q_{\frac{2}{3}}\right)} \leq C\left\|D u_{k}\right\|_{L^{p}\left(Q_{\frac{2}{3}}\right)}$.

Claim II: $u_{k}-U_{k} \rightarrow 0$ in $L^{2}\left(Q_{\frac{2}{3}}\right)$ and $L^{p}\left(Q_{\frac{2}{3}}\right)$.

Proof of Claim I: Let $(\alpha)_{k}=a_{k}\left(u_{k}, D u_{k}\right)_{h_{k}}$ and replace $\phi$ in the inequality for $u_{k}$ by $\phi_{-h_{k}}$, whose support is in $Q_{5 / 6}$ if $h_{k}$ is sufficiently small. From (4.7), the following holds

$$
\left|\iint_{Q_{1}}\left[-\left\langle g_{k}, \phi_{t}\right\rangle+\left\langle(\alpha)_{k}, D \phi\right\rangle\right] d z\right| \leq \varepsilon_{k}\left\|D u_{k}\right\|_{L^{p}\left(Q_{1}\right)}^{\frac{p}{q}}\|D \phi\|_{L^{p}\left(Q_{1}\right)}, \forall \phi \in C_{0}^{1}\left(Q_{1}\right) .
$$

For any $\tau \in\left(-\left(\frac{2}{3}\right)^{p}, 0\right)$ and sufficiently small positive $h$, let $\eta(s)$ be a $C^{1}$ function such that $\eta(s)=0$ if $s<\tau, \eta(s)=0$ if $s>\tau+h$ and $\eta$ is almost linear in $(\tau, \tau+h)$. Subtracting this inequality by the equation of $U_{k}$ and testing the result with $\left(U_{k}(x, s)-g_{k}(x, s)\right) \eta^{2}(s)$, which vanishes on the boundary of $Q_{\frac{2}{3}}$, we easily obtain the following for $\phi=U_{k}-g_{k}$ when we send $h$ to 0

$$
\sup _{s \in\left[-\frac{4}{9}, \tau\right]} \int_{B_{\frac{2}{3}}}|\phi|^{2} d x+\iint_{Q_{\frac{2}{3}}^{\tau}}\left\langle A_{k}\left(U_{k}, D U_{k}\right)-(\alpha)_{k}, D \phi\right\rangle d z \leq \varepsilon_{k}\left\|D u_{k}\right\|_{L^{p}\left(Q_{1}\right)}^{\frac{p}{q}}\|D \phi\|_{L^{p}\left(Q_{1}\right)} .
$$

Here, for any cylinder $Q$, we denote $Q^{t}=Q \cap\{(x, s): s \leq t\}$. The above then implies

$$
\begin{aligned}
& \sup _{s \in\left[-\frac{4}{9}, \tau\right]} \int_{B_{\frac{2}{3}}}|\phi|^{2} d x+\iint_{Q_{\frac{2}{3}}^{\tau}}\left\langle a\left(U_{k}, D U_{k}\right), D U_{k}\right\rangle d z= \\
& \quad \iint_{Q_{\frac{2}{3}}^{\tau}}\left\langle A_{k}\left(U_{k}, D U_{k}\right), D g_{k}\right\rangle+\left\langle(\alpha)_{k},\left(D U_{k}-D g_{k}\right)\right\rangle d z+\varepsilon_{k}\left\|D u_{k}\right\|_{L^{p}\left(Q_{1}\right)}^{\frac{p}{q}}\left\|D \phi_{h_{k}}\right\|_{L^{p}\left(Q_{1}\right)} .
\end{aligned}
$$

Since $\left\|D\left(U_{k}\right)_{h_{k}}\right\|_{L^{p}\left(Q_{1}\right)} \leq C\left\|D U_{k}\right\|_{L^{p}\left(Q_{1}\right)}$, a simple use of the Young inequality with $\varepsilon_{k}$ sufficiently small and the ellipticity of $A_{k}$ in a.1) give

$$
\begin{aligned}
\sup _{s \in\left[-\frac{4}{9}, \tau\right]} & \int_{B_{\frac{2}{3}}}\left|U_{k}-g_{k}\right|^{2} d x+\iint_{Q_{\frac{2}{3}}^{\tau}}\left|D U_{k}\right|^{p} d z \leq \\
& C \iint_{Q_{\frac{2}{3}}^{\tau}}\left[\left|D g_{k}\right|^{p}+\left|(\alpha)_{k}\right|^{q}+\left|D u_{k}\right|^{p}\right] d z+\varepsilon_{k}\left\|D u_{k}\right\|_{L^{p}\left(Q_{1}\right)}^{\frac{p}{q}}\left\|D \phi_{h_{k}}\right\|_{L^{p}\left(Q_{1}\right)} .
\end{aligned}
$$


Because $\left\|D g_{k}\right\|_{L^{p}\left(Q_{\frac{2}{3}}^{\tau}\right)} \leq C\left\|D u_{k}\right\|_{L^{p}\left(Q_{\frac{2}{3}}^{\tau}\right)}$ and $\left\|(\alpha)_{k}\right\|_{L^{q}\left(Q_{\frac{2}{3}}^{\tau}\right)}^{q}=\left\|A_{k}\left(u_{k}, D u_{k}\right)_{h_{k}}\right\|_{L^{q}\left(Q_{\frac{2}{3}}^{\tau}\right)}^{q} \leq$ $C\left\|D u_{k}\right\|_{L^{p}\left(Q_{\frac{2}{3}}^{\tau}\right)}^{p}$, we obtain from the above and the Young inequality the following estimate

$$
\left\|D U_{k}\right\|_{L^{p}\left(Q_{\frac{2}{3}}^{\tau}\right)}^{p} \leq C\left\|D u_{k}\right\|_{L^{p}\left(Q_{\frac{2}{3}}^{\tau}\right)}^{p}+\varepsilon_{k}\left\|D U_{k}\right\|_{L^{p}\left(Q_{\frac{2}{3}}\right)}^{p} \quad \forall \tau \in\left[-\left(\frac{2}{3}\right)^{p}, 0\right] .
$$

This established our first claim if we take $\tau=0$.

Proof of Claim II: Now, for any $\rho, r \in\left(0, \frac{2}{3}\right)$, we write $Q_{1}^{\prime}=Q_{\frac{2}{3}} \cap\{(x, s): s \leq$ $-\left(\frac{2}{3}\right)^{p}+\rho^{2}, Q_{2}^{\prime}=Q_{\frac{2}{3}} \cap\left\{(x, t):|x|>\frac{2}{3}-r\right\}$ and $Q_{3}^{\prime}=Q_{1}^{\prime} \cap Q_{2}^{\prime}$. These sets are the thin layers at the base and lateral sides of the cylinder $Q_{\frac{2}{3}}$.

Let $\phi(x, t)=\psi(x) \eta(t)$, where $\psi, \eta$ are respectively cut-off functions in $x, t$. That is, $\psi$ is a cut-off function for $B_{\frac{2}{3}-r}$ and $B_{\frac{2}{3}}$ and $\eta$ is a cut-off function for $\left[-\left(\frac{2}{3}\right)^{p}+\rho^{2}, 0\right]$ and $\left[-\left(\frac{2}{3}\right)^{p}, 0\right]$. We can assume that $|D \psi| \leq 1 / r$ and $\left|\eta_{t}\right| \leq 1 / \rho^{2}$.

Denote $H_{k}=u_{k}-U_{k}$ and $\Phi=H_{k} \phi^{2}$. Testing the equations for $U_{k}$ with $\Phi$ and replacing $\phi$ in (4.7) by $\Phi$, we get by subtracting the two results

$$
\left|\iint_{Q_{\frac{2}{3}}}\left[H_{k} \frac{\partial \Phi}{\partial t}+\left\langle A_{k}\left(u_{k}, D u_{k}\right)-A_{k}\left(U_{k}, D U_{k}\right), D \Phi\right\rangle\right] d z\right| \leq \varepsilon_{k}\left\|D u_{k}\right\|_{L^{p}\left(Q_{\frac{2}{3}}\right)}^{p / q}\|D \Phi\|_{L^{p}\left(Q_{\frac{2}{3}}\right)} .
$$

We now write $\mathbf{A}=A_{k}\left(u_{k}, D u_{k}\right)-A_{k}\left(U_{k}, D U_{k}\right)$ and derive from the above the following

$$
\begin{aligned}
& \sup _{s \in\left[-\left(\frac{2}{3}\right)^{p}, 0\right]} \int_{B_{\rho}} H_{k}^{2} \phi^{2} d x+\iint_{Q_{\frac{2}{3}}}\langle\mathbf{A}, D \Phi\rangle d z \leq \\
& \quad \iint_{Q}\left|H_{k}\right|^{2} \phi\left|\frac{\partial \phi}{\partial t}\right| d z+\varepsilon_{k}\left\|D u_{k}\right\|_{L^{p}\left(Q_{\frac{2}{3}}\right)}^{p / q}\|D \Phi\|_{L^{p}\left(Q_{\frac{2}{3}}\right)} .
\end{aligned}
$$

We consider the second term on the left and note that $D \Phi=\phi^{2} D H_{k}+2 H_{k} \phi D \phi$. By the monotonicity assumption a.4) with $u=u_{k}$ and $v=U_{k}$, we have

$$
\mathbf{A} D H_{k} \phi^{2} \geq \lambda_{0} \phi^{2}\left|D H_{k}\right|^{p} .
$$

On the other hand, because $D \phi=0$ in $Q \backslash Q_{2}^{\prime},|D \phi| \leq 1 / r$ and $|\mathbf{A}| \leq C\left[\left|D u_{k}\right|^{p-1}+\right.$ $\left.\left|D U_{k}\right|^{p-1}\right]$, we also have via the Young inequality

$$
\begin{aligned}
\iint_{Q_{\frac{2}{3}}} \mathbf{A} H_{k} \phi D \phi d z & \leq C \iint_{Q_{\frac{2}{3}}}\left(\left|D u_{k}\right|^{p-1}+\left|D U_{k}\right|^{p-1}\right)\left|H_{k}\right||\phi||D \phi| d z \\
& \leq C \iint_{Q_{2}^{\prime}}\left[\left|D u_{k}\right|^{p}+\left|D U_{k}\right|^{p}\right] d z+C \frac{1}{r^{p}} \iint_{Q_{2}^{\prime}} H_{k}^{p} d z .
\end{aligned}
$$

Also, because $\phi_{t}=0$ in $Q \backslash Q_{1}^{\prime}$ and $\left|\phi_{t}\right| \leq 1 / \rho^{2}$, we have

$$
\iint_{Q}\left|H_{k}\right|^{2} \phi\left|\frac{\partial \phi}{\partial t}\right| d z \leq C \frac{1}{\rho^{2}} \iint_{Q_{1}^{\prime}}\left|H_{k}\right|^{2} d z
$$

On the other hand, as

$$
\|D \Phi\|_{L^{p}\left(Q_{\frac{2}{3}}\right)}^{p} \leq C\left\|D H_{k}\right\|_{L^{p}\left(Q_{\frac{2}{3}}\right)}^{p}+C \frac{1}{r^{p}} \iint_{Q_{2}^{\prime}} H_{k}^{p} d z,
$$


a simple use of the Young inequality and the above estimates allow us to deduce from (4.13) that

$$
\sup _{s \in\left[-\left(\frac{2}{3}\right)^{p}, 0\right]} \int_{B_{\rho}} H_{k}^{2} \phi^{2} d x+\iint_{Q_{\frac{2}{3}}} \lambda_{0}\left|D H_{k}\right|^{p} \phi^{p} d z \leq F_{k}
$$

where

$$
\begin{gathered}
F_{k}=\quad \varepsilon_{k} \iint_{Q_{\frac{2}{3}}}\left(\left|D u_{k}\right|^{p}+\left|D U_{k}\right|^{p}\right) d z+C \iint_{Q_{2}^{\prime}}\left[\left|D u_{k}\right|^{p}+\left|D U_{k}\right|^{p}\right] d z+ \\
\quad C \frac{1}{r^{p}} \iint_{Q_{2}^{\prime}} H_{k}^{p} d z+C \frac{1}{\rho^{2}} \iint_{Q_{1}^{\prime}} H_{k}^{2} d z
\end{gathered}
$$

For any given $\varepsilon>0$ we will show that if $r, \rho$ are sufficiently small and $k$ is large then

$$
\frac{1}{r^{p}} \iint_{Q_{2}^{\prime}}\left|H_{k}\right|^{p} d z+\frac{1}{\rho^{2}} \iint_{Q_{1}^{\prime}}\left|H_{k}\right|^{2} d z<\varepsilon
$$

and

$$
\iint_{Q_{2}^{\prime}}\left(\left|D u_{k}\right|^{p}+\left|D U_{k}\right|^{p}\right) d z<\varepsilon
$$

The above estimates yield

$$
\sup _{s \in\left[-\left(\frac{2}{3}\right)^{p}, 0\right]} \int_{B_{\rho}} H_{k}^{2} d x+\iint_{Q_{\frac{2}{3}}} \lambda_{0}\left|D H_{k}\right|^{p} \phi^{p} d z \leq C \varepsilon_{k}+2 \varepsilon
$$

and thus $\left\|H_{k}\right\|_{L^{2}\left(Q_{\frac{1}{2}}\right)}<\varepsilon$. By Sobolev's imbedding inequality (see [1, Proposition 3.1, p.7]), we also have $\left\|H_{k}\right\|_{L^{p}\left(Q_{\frac{1}{2}}\right)}<\varepsilon$. Choosing $\varepsilon$ small we obtain the desired contradiction.

Concerning (4.16), when $r, \rho$ have been fixed, because $H_{k}=\left(u_{k}-g_{k}\right)+\left(g_{k}-U_{k}\right)$ and $u_{k}-g_{k}$ converge to 0 in $L^{p}$ and $L^{2}$, we need only to prove that

$$
\frac{1}{r^{p}} \iint_{Q_{2}^{\prime}}\left|g_{k}-U_{k}\right|^{p} d z+\frac{1}{\rho^{2}} \iint_{Q_{1}^{\prime}}\left|g_{k}-U_{k}\right|^{2} d z<\varepsilon
$$

if $k$ is large and $r, \rho$ are sufficiently small (uniformly in $k$ ).

For the integral on $Q_{1}^{\prime}$, from (4.11), we find that

$$
\begin{array}{r}
\iint_{Q_{1}^{\prime}}\left|U_{k}-g_{k}\right|^{2} d z \leq C \rho^{2} \sup _{s \in\left[-\left(\frac{2}{3}\right)^{p},-\left(\frac{2}{3}\right)^{p}+\rho^{2}\right]} \int_{B_{\frac{2}{3}, s}}\left|U_{k}-g_{k}\right|^{2} d x \leq \\
C \rho^{2} \iint_{Q_{1}^{\prime}}\left[\left|D g_{k}\right|^{p}+\left|(\alpha)_{h_{k}}\right|^{q}\right] d z+\varepsilon_{k} \rho^{2}\left\|D U_{k}\right\|_{L^{p}\left(Q_{\frac{2}{3}}\right)}^{p}
\end{array}
$$

On the other hand, because $U_{k}=g_{k}$ on the lateral part of $S_{\frac{2}{3}}$, we can use the Poincaré inequality in $x$ to get

$$
\frac{1}{r^{p}} \iint_{Q_{2}^{\prime}}\left|U_{k}-g_{k}\right|^{p} d z \leq \iint_{Q_{2}^{\prime}}\left|D U_{k}-D g_{k}\right|^{p} d z \leq \iint_{Q_{2}^{\prime}}\left|D g_{k}\right|^{p}+\left|D U_{k}\right|^{p} d z .
$$


By (4.11) again,

$$
\begin{aligned}
\iint_{Q_{2}^{\prime}}\left|D U_{k}\right|^{p} d z & \leq \iint_{Q_{2}^{\prime} \backslash Q_{1}^{\prime}}\left|D U_{k}\right|^{p} d z+\iint_{Q_{1}^{\prime}}\left|D U_{k}\right|^{p} d z \\
& \leq \iint_{Q_{2}^{\prime} \backslash Q_{1}^{\prime}}\left|D U_{k}\right|^{p} d z+\iint_{Q_{1}^{\prime}}\left[\left|D g_{k}\right|^{p}+\left|(\alpha)_{h_{k}}\right|^{q}\right] d z
\end{aligned}
$$

But

$$
\iint_{Q_{1}^{\prime}}\left[\left|D g_{k}\right|^{p}+\left|(\alpha)_{h_{k}}\right|^{q}\right] d z \leq C \iint_{Q_{1}^{\prime}}\left|D u_{k}\right|^{p} d z
$$

Therefore, the left hand side of (4.18) can be estimated by

$$
C\left[\iint_{Q_{1}^{\prime}}\left|D u_{k}\right|^{p} d z+\iint_{Q_{2}^{\prime}}\left|D u_{k}\right|^{p} d z+\iint_{Q_{2}^{\prime} \backslash Q_{1}^{\prime}}\left|D U_{k}\right|^{p} d z+\varepsilon_{k}\right] .
$$

Obviously, the left hand side of (4.17) is also bounded by the above. Thus, we need only prove that the integrals in (4.19) can be arbitrarily small (uniformly in $k$ ) if $r, \rho$ are sufficiently small. By the uniform continuity of integrals (see Lemma 3.10), the integral of $\left|D u_{k}\right|^{p}$ over $Q_{1}^{\prime}$ is small if the measure $\left|Q_{1}^{\prime}\right|$, or $\rho$, is sufficiently small (but independent of $k$ ). Hence, the first term of (4.19) is small. Fixing such a $\rho$, We then repeat the argument to see that if $r$ is small then so is the second integral in (4.19). Similarly, the integral of $\left|D U_{k}\right|^{p}$ over $Q_{2}^{\prime} \backslash Q_{1}^{\prime}$ is small.

Therefore, the right hand side $F_{k}$ of (4.14) can be arbitrarily small if $r, \rho$ are sufficiently small and $k$ is large. As we mentioned earlier this gives the proof of the second claim and completes our proof.

We now consider the following alternative of the monotonicity condition a.4).

a.4') For any $w \in \mathbb{R}^{m}$ and $U, V \in \mathbb{R}^{n m}$, there holds

$$
\langle A(w, U)-A(w, V), U-V\rangle \geq \lambda_{0}|U-V|^{2} \begin{cases}\min \left\{|U|^{p-2},|V|^{p-2}\right\} & U \neq 0 \text { or } V \neq 0 \\ 0 & \text { otherwise. }\end{cases}
$$

Moreover, $A(u, \zeta)$ is Lipschitz in $u$ in the following sense

$$
|A(u, \zeta)-A(v, \zeta)| \leq C|u-v \| \zeta|^{p-1} .
$$

Concerning the condition (4.20), if $A(u, U)$ is differentiable in $U$ then we note that

$$
\langle A(w, U)-A(w, V), U-V\rangle=\int_{0}^{1}\left\langle\frac{\partial A}{\partial \zeta}(w, s U+(1-s) V) d s(U-V), U-V\right\rangle .
$$

Therefore, (4.20) can be verified if the matrix $\frac{\partial A}{\partial \zeta}$ is positive definite and

$$
\left\langle\frac{\partial A}{\partial \zeta}(w, s U+(1-s) V) \eta, \eta\right\rangle \geq \lambda(s U+(1-s) V)|\eta|^{2} \quad \forall s \in(0,1), \eta \in \mathbb{R}^{n m}
$$

for some $\lambda(s U+(1-s) V) \geq|s U+(1-s) V|^{p-2}$. 
Proposition 4.2 The conclusion of Proposition 4.1 holds if the monotonicity condition a.4) is replaced by $a .4^{\prime}$ ').

Proof: We revisit the proof of Proposition 4.1 and point out necessary modifications under a.4'). As before, for $H_{k}=u_{k}-U_{k}$ and $\phi(x, t)=\psi(x) \eta(t)$ with $\psi, \eta$ being respectively cut-off functions in $x, t$ for $Q_{\frac{2}{3}}$. That is, $\psi$ is a cut-off function for $B_{\frac{2}{3}-r}$ and $B_{\frac{2}{3}}$ and $\eta$ is a cut-off function for $\left[-\left(\frac{2}{3}\right)^{p}+\rho^{2}, \tau\right]$ and $\left[-\left(\frac{2}{3}\right)^{p}, \tau\right]$, where $\tau$ is any number in $\left[-\left(\frac{2}{3}\right)^{p}, 0\right]$.

Again, the proof is by contradiction and we can see that the proof of claim I in the proof of Proposition 4.1 is still applicable here. We need only consider claim II. First of all, the assumptions (4.8) and (4.9) give that

$$
\iint_{Q_{\frac{2}{3}}}\left|H_{k}\right|^{2} d z \geq \varepsilon_{1}>0
$$

for some fixed $\varepsilon_{1}$. So that, if $r \geq 2, s>1$ then Hölder inequality, the boundedness of $H_{k}$ and the above inequality yield

$$
\left(\iint_{Q_{\frac{2}{3}}}\left|H_{k}\right|^{r} d z\right)^{\frac{1}{s}} \leq C(M)\left(\iint_{Q_{\frac{2}{3}}}\left|H_{k}\right|^{2} d z\right)^{\frac{1}{s}} \leq C\left(M, r, s, \varepsilon_{1}\right) \iint_{Q_{\frac{2}{3}}}\left|H_{k}\right|^{2} d z .
$$

Since a.4) was not used until we obtain (4.13), we now need only look at the integral of $\left\langle\mathbf{A}, D H_{k} \phi^{2}\right\rangle$ in (4.13), which reads

$$
\sup _{s \in\left[-\left(\frac{2}{3}\right)^{p}, 0\right]} \int_{B_{\frac{2}{3}}} H_{k}^{2} \phi^{2} d x+\iint_{Q_{\frac{2}{3}}}\left\langle\mathbf{A}, D H_{k} \phi^{2}\right\rangle d z \leq F_{k},
$$

where $\mathbf{A}=A_{k}\left(u_{k}, D u_{k}\right)-A_{k}\left(U_{k}, D U_{k}\right)$, and $F_{k}=F_{k}(h, r, \rho)$ which is defined by (4.15) and can be arbitrarily small if $k$ is large and $h, r, \rho$ small (uniformly in $k$ ) thanks to the argument in the proof of Proposition 4.1 without using a.4).

We now consider the following two cases.

The case $p>2$ : We write $Q_{\frac{2}{3}}=E_{u} \cup E_{v}$ where

$$
E_{u}=\left\{z:\left|D u_{k}(z)\right| \leq\left|D U_{k}(z)\right|\right\}, E_{v}=\left\{z:\left|D U_{k}(z)\right|<\left|D u_{k}(z)\right|\right\} .
$$

We also write

$$
\iint_{Q_{\frac{2}{3}}}\left\langle\mathbf{A}, D H_{k} \phi^{2}\right\rangle d z=\iint_{E_{u}}\left\langle\mathbf{A}, D H_{k} \phi^{2}\right\rangle d z+\iint_{E_{v}}\left\langle\mathbf{A}, D H_{k} \phi^{2}\right\rangle d z .
$$

On $E_{u}$, we have

$$
\left\langle\mathbf{A}, D H_{k}\right\rangle=\left\langle A_{k}\left(U_{k}, D U_{k}\right)-A_{k}\left(U_{k}, D u_{k}\right)+A_{k}\left(U_{k}, D u_{k}\right)-A_{k}\left(u_{k}, D u_{k}\right), D\left(U_{k}-u_{k}\right)\right\rangle .
$$

By (4.20), as $\left|D u_{k}(z)\right| \leq\left|D U_{k}(z)\right|$ and $p>2$, it follows that

$$
\left\langle A_{k}\left(U_{k}, D U_{k}\right)-A_{k}\left(U_{k}, D u_{k}\right), D U_{k}-D u_{k}\right\rangle \geq \lambda_{0}\left|D u_{k}\right|^{p-2}\left|D U_{k}-D u_{k}\right|^{2}
$$


and this term will be kept on the left of (4.22). On the other hand, as $A_{k}(u, \zeta)$ is Lipschitz in $u$,

$$
\begin{aligned}
& \left|\left\langle A_{k}\left(U_{k}, D u_{k}\right)-A_{k}\left(u_{k}, D u_{k}\right), D H_{k}\right\rangle\right| \leq C\left|H_{k}\right|\left|D u_{k}\right|^{p-1}\left|D H_{k}\right| \\
& =C\left|H_{k}\right|\left|D u_{k}\right|^{\frac{p}{2}}\left|D u_{k}\right|^{\frac{p-2}{2}}\left|D H_{k}\right| \leq \frac{\lambda_{0}}{4}\left|D u_{k}\right|^{p-2}\left|D H_{k}\right|^{2}+\left.C\left|H_{k}\right|^{2}|| D u_{k}\right|^{p} .
\end{aligned}
$$

The first term on the right can be absorbed into (4.23).

Interchange the roles of $u_{k}, U_{k}$ and apply a similar treatment for the integral of $\left\langle\mathbf{A}, D H_{k} \phi^{2}\right\rangle$ over $E_{v}$ in the above argument to see that (4.22) gives

$$
\begin{array}{r}
\sup _{s \in\left[-\left(\frac{2}{3}\right)^{p}, 0\right]} \int_{B_{\frac{2}{3}}} H_{k}^{2} \phi^{2} d x+\iint_{E_{u}}\left|D u_{k}\right|^{p-2}\left|D H_{k}\right|^{2} \phi^{2} d z+\iint_{E_{v}}\left|D U_{k}\right|^{p-2}\left|D H_{k}\right|^{2} \phi^{2} d z \\
\leq C \iint_{E_{u}}\left|D u_{k}\right|^{p}\left|H_{k}\right|^{2} \phi^{2} d z+C \iint_{E_{u}}\left|D U_{k}\right|^{p}\left|H_{k}\right|^{2} \phi^{2} d z+F_{k} .
\end{array}
$$

Since $\left|D u_{k}\right| \leq\left|D U_{k}\right|$ on $E_{u}$, the above yields

$$
\sup _{s \in\left[-\left(\frac{2}{3}\right)^{p}, 0\right]} \int_{B_{\frac{2}{3}}} H_{k}^{2} \phi^{2} d x \leq C \iint_{Q_{\frac{2}{3}}}\left|D U_{k}\right|^{p}\left|H_{k}\right|^{2} \phi^{2} d z+F_{k}
$$

Now, using the higher integrability of $D u_{k}$ of Lemma 3.11 we also have the $L^{q}$ estimate, with $q>p$, for $D U_{k}$ on $Q_{\frac{2}{3}}$ by extending $U_{k}=u_{k}$ beyond the boundary of $Q_{\frac{2}{3}}$. In fact, this comes from (4.12) (for cylinders centered on the boundary of $Q_{\frac{2}{3}}$ ) to obtain a reverse Hölder inequality for $D U_{k}$ and then use the fact that $D u_{k}$ is in $L^{q}$. Thus, by Hölder's inequality and (4.21)

$$
\iint_{Q_{\frac{2}{3}}}\left|D U_{k}\right|^{p}\left|H_{k}\right|^{2} \phi^{2} d z \leq C(M)\left(\iint_{Q_{\frac{2}{3}}}\left|H_{k}\right|^{r} \phi^{r} d z\right)^{\frac{1}{s}} \leq C\left(\varepsilon_{1}, M\right) \iint_{Q_{\frac{2}{3}}^{\tau}}\left|H_{k}\right|^{2} d z .
$$

Again, it is easy to see that the above argument still holds if $Q_{\frac{2}{3}}$ is replace by $Q_{\frac{2}{3}}^{\tau}=$ $Q_{\frac{2}{3}} \cap\{(x, t): t \leq \tau\}$ for any $\tau \leq 0$. Sending $\rho, r$ to zero to obtain

$$
\sup _{s \in\left[-\left(\frac{2}{3}\right)^{p}, \tau\right]} \int_{B_{\frac{2}{3}}} H_{k}^{2} d x \leq C\left(\varepsilon_{1}, M\right) \iint_{Q_{\frac{2}{3}}^{\tau}}\left|H_{k}\right|^{2} d z+F_{k} .
$$

Setting

$$
y_{k}(\tau)=\sup _{s \in\left[-\left(\frac{2}{3}\right)^{p}, \tau\right]} \int_{B_{\frac{2}{3}}} H_{k}^{2} d x
$$

the above (4.24) becomes $y_{k}^{\prime} \leq C y_{k}+F_{k}$ with $y\left(-\left(\frac{2}{3}\right)^{p}\right), F_{k}$ can be arbitrarily small. By the Gronwall inequality, we see that $H_{k} \rightarrow 0$ in $L^{2}\left(Q_{\frac{2}{3}}\right)$ as well as in $L^{p}\left(Q_{\frac{2}{3}}\right)$ because $p>2$ and $H_{k}$ is bounded. Our desired contradiction is obtained and the proof is complete for the case $p>2$.

The case $2 n /(n+2)<p<2$ : For each $z \in Q$, let us denote by $u(z), v(z)$ which are either $u_{k}$ or $U_{k}$ such that, with a slight abuse of notation here, $D u(z)=\max \left\{D u_{k}(z), D U_{k}(z)\right\}$ and $D v(z)=\min \left\{D u_{k}(z), D U_{k}(z)\right\}$. We write $Q_{\frac{2}{3}}=E_{u} \cup E_{v}$ where

$$
E_{u}=\left\{z: \frac{1}{2}|D u(z)| \leq|D u(z)-D v(z)|\right\}, E_{v}=\left\{z:|D u(z)-D v(z)|<\frac{1}{2}|D u(z)|\right\} .
$$


Again, we consider the integral of $\left\langle\mathbf{A}, D H_{k} \phi^{2}\right\rangle$ in (4.22). On $E_{u}$, we write $H=u-v$, $D H=D u-D v$ and

$$
\left\langle\mathbf{A}, D H_{k}\right\rangle=\left\langle A_{k}(u, D u)-A_{k}(u, D v)+A_{k}(u, D v)-A_{k}(v, D v), D H\right\rangle .
$$

Because $p<2, \min \left\{\left|D u_{k}\right|^{p-2},\left|D u_{k}\right|^{p-2}\right\}=|D u|^{p-2}$. By (4.20) and the fact that $|D u|^{p-2} \geq$ $2^{p-2}|D v-D u|^{p-2}$, we have

$$
\left\langle A_{k}(u, D u)-A_{k}(u, D v), D u-D v\right\rangle \geq C \lambda_{0}|D v-D u|^{p},
$$

and this term will be kept on the left of (4.22). On the other hand, as $A_{k}(v, \zeta)$ is Lipschitz in $v$

$$
\begin{aligned}
\left|\left\langle A_{k}(u, D v)-A_{k}(v, D v), D H\right\rangle\right| & \leq C|H||D v|^{p-1}|D H| \leq \frac{C \lambda_{0}}{4}|D H|^{p}+\left.C|H|^{\frac{p}{p-1}}|| D v\right|^{p} \\
& \leq \frac{C \lambda_{0}}{4}|D H|^{p}+\left.C(M)|H|^{2}|| D v\right|^{p} .
\end{aligned}
$$

Here, we have used the fact that $H$ is bounded in the last inequality. The first term on the right can be absorbed into (4.25).

On $E_{v}$ we note that $|D v-D u|<\frac{1}{2}|D u|$ implies $|D v| \geq \frac{1}{2}|D u|$ (otherwise, $|D v-D u| \geq$ $|D u|-|D v|>\frac{1}{2}|D u|$ contradicting the definition of $\left.E_{v}\right)$. We now write $H=v-u$, $D H=D v-D u$ and

$$
\left\langle\mathbf{A}, D H_{k}\right\rangle=\left\langle A_{k}(v, D v)-A_{k}(v, D u)+A_{k}(v, D u)-A_{k}(u, D u), D H\right\rangle .
$$

We have by (4.20)

$$
\left\langle A_{k}(v, D u)-A_{k}(v, D v), D v-D u\right\rangle \geq C \lambda_{0}|D u|^{p-2}|D v-D u|^{2} .
$$

Again, this term will stay on the left of (4.22). Meanwhile, as $|D v| \sim|D u|$ on $E_{v}$, we have

$$
\begin{aligned}
& \mid\left\langle A_{k}(v, D u)-\right.\left.A_{k}(u, D u), D H\right\rangle\left.|\leq C| H|| D u\right|^{p-1}|D H| \leq \\
& C|H||D v|^{\frac{p}{2}}|D u|^{\frac{p-2}{2}}|D H| \leq \frac{C \lambda_{0}}{4}|D u|^{p-2}|D H|^{2}+C|H|^{2}|D v|^{p} .
\end{aligned}
$$

Combining the above estimates and noting that $|D v| \leq\left|D U_{k}\right|$, we derive from (4.22)

$$
\begin{aligned}
\sup _{s \in\left[-\left(\frac{2}{3}\right)^{p}, 0\right]} \int_{B_{\frac{2}{3}}} H_{k}^{2} \phi^{2} d x & +\iint_{E_{u}}\left|D H_{k}\right|^{p} \phi^{2} d z+\iint_{E_{v}}|D u|^{p-2}\left|D H_{k}\right|^{2} \phi^{2} d z \\
& \leq C(M) \iint_{Q_{\frac{2}{3}}}\left|D U_{k}\right|^{p}\left|H_{k}\right|^{2} \phi^{2} d z+F_{k} .
\end{aligned}
$$

As in the case $p>2$, because $2 n /(n+2)<p<2$ the higher integrability of $D U_{k}$ is available, we can derive a Gronwall inequality and see that $H_{k} \rightarrow 0$ in $L^{2}\left(Q_{\frac{2}{3}}\right)$. Since $H_{k}$ is bounded and $D U_{k}$ is $L^{q}\left(Q_{\frac{2}{3}}\right)$, an application of Hölder's inequality shows that the right hand side of the above inequality tends to zero as $k \rightarrow \infty$. Hence,

$$
\iint_{E_{u}}\left|D H_{k}\right|^{p} \phi^{2} d z+\iint_{E_{v}}|D u|^{p-2}\left|D H_{k}\right|^{2} \phi^{2} d z \rightarrow 0 \quad \text { as } k \rightarrow \infty .
$$


Concerning the $L^{p}$ norm of $D H_{k}$, we observe that

$$
\begin{aligned}
\iint_{E_{v}}\left|D H_{k}\right|^{p} \phi^{2} d z & =\iint_{E_{v}}|D u|^{\frac{(p-2) p}{2}}\left|D H_{k}\right|^{p}|D u|^{\frac{(2-p) p}{2}} \phi^{2} d z \\
& \leq\left(\iint_{E_{v}}|D u|^{p-2}\left|D H_{k}\right|^{2} \phi^{2} d z\right)^{\frac{p}{2}}\left(\iint_{E_{v}}|D u|^{p} \phi^{2} d z\right)^{\frac{2-p}{2}} .
\end{aligned}
$$

Because the integral of $|D u|^{p}=\max \left\{\left|D u_{k}\right|^{p},\left|D U_{k}\right|^{p}\right\}$ over $Q_{\frac{2}{3}}$ is bounded, the above and (4.27) show that the integral of $\left|D H_{k}\right|^{p}$ over $E_{u}$ and $E_{v}$, and therefore $Q_{\frac{2}{3}}$, tends to zero. By Sobolev's imbedding inequality, we see that $H_{k} \rightarrow 0$ in $L^{p}\left(Q_{\frac{2}{3}}\right)$. Our proof is then complete.

The first alternative (4.4) in the above propositions is not as useful as (4.6) for our later proof of the Hölder continuity for weak solutions. To this end, we will show that (4.4) allows us to approximate the considered $u$ by solutions to those of nice systems whose coefficients $A(v, D v)$ do not involve with the solutions $v$. The proof of this fact for the singular case $(p<2)$ is much more involved and will be reported in a forthcoming work. Here, we present only the result when $p>2$.

Proposition 4.3 Assume that $p>2$ and a.1)-a.3) and a.4) or a.4') hold. For any given $M, \varepsilon>0$ and $\beta>1$ there exists $\delta \in(0,1]$ that depends only on $\lambda, \Lambda, \Lambda_{0}, \varepsilon, \beta$ such that if $A \in \mathcal{A}, B \in \mathcal{B}$ and $u \in V\left(Q_{R}\right)$ satisfying

$$
\oiiint_{Q_{R}}\left|u-u_{R}\right|^{2} d z+R^{p} \oiiint_{Q_{R}}|D u|^{p} d z \leq M,
$$

and

$$
\left|\iint_{Q_{R}}-u \phi_{t}+\langle A(u, D u), D \phi\rangle-\langle B(u, D u), \phi\rangle d z\right| \leq \delta\|D u\|_{L^{\frac{p}{q}\left(Q_{R}\right)}}\|D \phi\|_{L^{p}\left(Q_{R}\right)}
$$

for any $Q_{R} \subset Q, \phi \in V_{p}^{0}(Q)$, then there exists $v$ in $V_{p}\left(Q_{R / 2}\right)$ such that $\|v\|_{L^{\infty}\left(Q_{R / 2}\right)} \leq$ $C\left(\lambda, \Lambda, \lambda_{0}, M\right)$ and

$$
\left\{\begin{array}{l}
\oiiint_{Q_{R / 2}}|v-u|^{2} d z+\oiiint_{Q_{R / 2}}|v-u|^{p} d z \leq \varepsilon R^{p} \oiiint_{Q_{R}}|D u|^{p} d z \\
\oiiint_{Q_{R / 2}}|D v|^{p} d z \leq \beta \oiiint_{Q_{R / 2}}|D u|^{p} d z
\end{array}\right.
$$

Moreover, $v$ satisfies

$$
\iint_{Q_{R / 2}}-v \phi_{t}+\langle\widetilde{A}(v, D v), D \phi\rangle-\langle\widetilde{F}(v, D v), \phi\rangle d z=0, \quad \forall \phi \in C_{0}^{1}\left(Q_{R / 2}\right),
$$

where either $\widetilde{A}(v, D v)=A(v, D v)$ and $\widetilde{B}(v, D v)=B(v, D v)$ or $\widetilde{A}(v, D v)=A(c, D v)$ and $\widetilde{B}(v, D v)=B(c, D v)$ for some constant vector $c \in \mathbb{R}^{m}$.

Proof: Again, we will only discuss the case $B \equiv 0$ here. As in the proof of Proposition 4.2 , by scaling we can assume that $R=1$ and need only consider the case when

$$
\limsup _{k \rightarrow \infty} f_{Q_{1}}\left|D u_{k}\right|^{p} d z=0 \text {. }
$$


We now look at the solution of

$$
\iint_{Q_{\frac{2}{3}}}\left[-v_{k} \phi_{t}+A_{k}\left(\left(u_{k}\right)_{Q_{1}}, D v_{k}\right) D \phi\right] d z=0 \quad \forall \phi \in V_{p}^{0}\left(Q_{\frac{2}{3}}\right),
$$

and $v_{k}=u_{k}$ on $S_{\frac{2}{3}}$. By testing (4.33) with $v_{k}-u_{k}$ we easily see that

$$
\oiiint_{Q_{\frac{2}{3}}}\left|D v_{k}\right|^{p} d z \leq C \int_{Q_{1}}\left|D u_{k}\right|^{p} d z .
$$

As before, by subtracting the equations for $u_{k}, v_{k}$ and testing the result with $\left(v_{k}-u_{k}\right)$, we have for $H_{k}=v_{k}-u_{k}$ the following

$$
\sup _{t} \int_{B_{\frac{2}{3}}} H_{k}^{2} d x+\iint_{Q_{\frac{2}{3}}}\left\langle A_{k}\left(\left(u_{k}\right)_{Q_{1}}, D v_{k}\right)-A_{k}\left(u_{k}, D u_{k}\right), D H_{k}\right\rangle d z \leq 0 .
$$

We now write

$$
\begin{aligned}
A_{k}\left(\left(u_{k}\right)_{Q_{1}}, D v_{k}\right)-A_{k}\left(u_{k}, D u_{k}\right)= & A_{k}\left(\left(u_{k}\right)_{Q_{1}}, D v_{k}\right)-A_{k}\left(\left(u_{k}\right)_{Q_{1}}, D u_{k}\right) \\
& +A_{k}\left(\left(u_{k}\right)_{Q_{1}}, D u_{k}\right)-A_{k}\left(u_{k}, D u_{k}\right)
\end{aligned}
$$

and keep the first difference on the left of (4.35). Using the ellipticity and Lipschitz property of $A_{k}$, we obtain

$$
\sup _{t} \int_{B_{\frac{2}{3}}} H_{k}^{2} d x \leq C \iint_{Q_{\frac{2}{3}}}\left|D u_{k}\right|^{p}\left|u_{k}-\left(u_{k}\right)_{Q_{1}}\right|^{2} d z
$$

We now make use of the $L^{q}$ estimate for $D u_{k}$, see (3.23) of Lemma 3.11and Remark 3.12, to find some $q>p$ and $\sigma \geq 1$ such that

$$
\left(f_{Q_{\frac{2}{3}}}\left|D u_{k}\right|^{q} d z\right)^{\frac{p}{q}} \leq C\left(\oiiint_{Q_{1}}\left|D u_{k}\right|^{p} d z\right)^{\sigma} .
$$

Hence, for $r=(p / q)^{\prime}$

$$
\sup _{t} \int_{B_{\frac{2}{3}}}\left|H_{k}\right|^{2} d x \leq C\left(\iint_{Q_{1}}\left|D u_{k}\right|^{p} d z\right)^{\sigma}\left(\iint_{Q_{\frac{2}{3}}}\left|u_{k}-\left(u_{k}\right)_{Q_{1}}\right|^{2 r} d z\right)^{\frac{1}{r}} .
$$

Since $u_{k}$ is bounded,

$$
\left(\iint_{Q_{\frac{2}{3}}}\left|u_{k}-\left(u_{k}\right)_{Q_{1}}\right|^{2 r} d z\right)^{\frac{1}{r}} \leq C(M)\left(\iint_{Q_{\frac{2}{3}}}\left|u_{k}-\left(u_{k}\right)_{Q_{1}}\right|^{2} d z\right)^{\frac{1}{r}}
$$

By (4.32) and an application of the Poincaré inequality, we see that the above quantities tend to 0 as $k \rightarrow \infty$. Sending $\rho, \tau$, the parameters defining $\phi$, to 0 in (4.37) and using the boundedness of the integral of $\left|D u_{k}\right|^{p}$, we derive

$$
\sup _{t} \int_{B_{\frac{2}{3}}}\left|H_{k}\right|^{2} d x \leq \varepsilon \iint_{Q_{1}}\left|D u_{k}\right|^{p} d z
$$


for any given $\varepsilon>0$ when $k$ is sufficiently large. Integrating the above in $t$, we obtain

$$
\iint_{Q_{\frac{2}{3}}}\left|u_{k}-v_{k}\right|^{2} d z \leq \varepsilon \iint_{Q_{1}}\left|D u_{k}\right|^{p} d z
$$

Finally, by applying the interpolation inequality in $x$ to $\left(u_{k}-v_{k}\right)$, one has the following

$$
\int_{B_{\frac{2}{3}}}\left|u_{k}-v_{k}\right|^{p} d x \leq \varepsilon \int_{B_{\frac{2}{3}}}\left|D u_{k}-D v_{k}\right|^{p} d x+C(\varepsilon)\left(\int_{B_{\frac{2}{3}}}\left|u_{k}-v_{k}\right|^{2} d x\right)^{\frac{p}{2}} .
$$

Integrating with respect to $t$ and using (4.34) to get

$$
\iint_{Q_{\frac{2}{3}}}\left|u_{k}-v_{k}\right|^{p} d z \leq C \varepsilon \iint_{Q_{1}}\left|D u_{k}\right|^{p} d z+C(\varepsilon)\left(\sup _{t} \int_{B_{\frac{2}{3}}}\left|u_{k}-v_{k}\right|^{2} d x\right)^{\frac{p}{2}} .
$$

By (4.38), we see that

$$
\iint_{Q_{\frac{2}{3}}}\left|u_{k}-v_{k}\right|^{p} d z \leq C \varepsilon \iint_{Q_{1}}\left|D u_{k}\right|^{p} d z
$$

The proof is now complete by rescaling.

\section{Decay estimates and the proof of Theorem 2.2}

We will prove in this section that the set $\mathcal{I}$ of parameters with which the scaling decay property D) holds for our systems is open. Firstly, we recall the property D): For a given bounded function $v$ in $V_{p}\left(Q_{1,1}\right)$ we say that $v$ satisfies a scaling decay property if the following holds

D) Let $M=\sup _{Q_{1,1}}|v|$. For any $R_{0}>0$ and $\eta \in(0,1)$, there are positive numbers $A, K, L, \alpha_{0}, \omega_{0}$ depending on $M, \eta$ (with $K, A$ sufficiently large) such that we can define the following sequences

$$
R_{k}=\frac{R_{0}}{K^{k}}, \quad \omega_{k+1}=\max \left\{\eta \omega_{k}, L R_{n}^{\alpha_{0}}\right\}, \quad S_{k}=\frac{\omega_{k}}{A}, \quad Q_{k}=B_{R_{k}} \times\left[-S_{k}^{2-p} R_{k}^{p}, 0\right]
$$

such that $Q_{k} \subset Q_{1,1}$ for any integer $k \geq 0$ and

$$
\omega_{k}^{p} \geq \oiiint_{Q_{k}}\left|v-(v)_{k}\right|^{p} d z, \quad(v)_{k}=\oiiint_{Q_{k}} v d z .
$$

We then consider a family of systems of the form $(\tau \in[0,1])$

$$
\begin{cases}v_{t}=\operatorname{div}(A(\tau, v, D v)) & \text { in } Q_{1,1} \\ v=g & \text { on } S_{1,1}\end{cases}
$$


We defined $\mathcal{I}$ to be the collection of $\tau \in[0,1]$ such that every bounded weak solutions of the above system verifies $\mathrm{D}$ ). Theorem 2.2 asserted that $\mathcal{I}$ is open and bounded weak solutions to (5.3) with $\tau \in \mathcal{I}$ are Hölder continuous. Its proof goes as follows.

Proof of Theorem 2.2; Fix a $\mu \in \mathcal{I}$. We will show that if $|\nu-\mu|$ is sufficiently small then $\nu \in \mathcal{I}$. That is, every bounded weak solution $u$ of (5.3) with $\tau=\nu$ will satisfy D). Now, let $u$ be such a solution and $M=\sup _{Q}|u|$.

The new set of parameters $A, K, L, \alpha_{0}, R_{0},\left\{\omega_{k}\right\}$ in D) for $u$ will be determined in the course of our calculation and depend from that of the reference system (5.3) when $\tau=\mu$.

By induction, let us start with a positive $\omega_{k}$, says $k=0$, such that

$$
\omega_{k}^{p} \geq \oiiint_{Q_{k}}\left|u-(u)_{k}\right|^{p} d z .
$$

In the sequel, for any $t>0$ we will denote by $t Q_{k}$ the cylinder with radius $t R_{k}$ and concentric with $Q_{k}$. From the Caccioppoli inequality, see Lemma 3.3 with $\rho=S_{k}^{2-p} R_{k}^{p}$,

$$
R_{k}^{p} \int_{\frac{1}{2} Q_{k}}|D u|^{p} d z \leq C \oiiint_{Q_{k}}\left|u-(u)_{k}\right|^{p} d z+C \oiiint_{Q_{k}} S_{k}^{p-2}\left|u-(u)_{k}\right|^{2} d z .
$$

An application of Young's inequality to the integrand in the second term on the right and (5.4) we can find a constant $C_{1}$ such that

$$
R_{k}^{p} \oiiint_{\frac{1}{2} Q_{k}}|D u|^{p} d z \leq C \oiiint_{Q_{k}}\left|u-(u)_{k}\right|^{p} d z+C S_{k}^{p} \leq C_{1}\left(1+\frac{1}{A^{p}}\right) \omega_{k}^{p} .
$$

For any given $\varepsilon>0$, if $|\mu-\nu|$ is sufficiently small (depending only on $M, \varepsilon$ ) we apply the approximation result, Proposition 4.3, in $\frac{1}{2} Q_{k}$ to obtain a "nice" solution $v$ satisfying (5.3) with $\tau=\mu$ or a similar system with $v$ being replaced by a constant vector such that $\sup _{\frac{1}{4} Q_{k}}|v| \leq C(M)$ and (in combination with (5.4) and (5.5))

$$
\begin{aligned}
\oiiint_{\frac{1}{4} Q_{k}}\left|v-(v)_{k}\right|^{p} d z & \leq \oiiint_{\frac{1}{4} Q_{k}}\left|v-(u)_{k}\right|^{p} d z \\
& \leq \oiiint_{\frac{1}{4} Q_{k}}|v-u|^{p} d z+\oiiint_{\frac{1}{4} Q_{k}}\left|u-(u)_{k}\right|^{p} d z \\
& \leq \varepsilon R_{k}^{p} \int_{\frac{1}{2} Q_{k}}|D u|^{p} d z+C_{2} \omega_{k}^{p} \leq C_{3}\left(1+\frac{1}{A^{p}}\right) \omega_{k}^{p} \leq C_{4} \omega_{k}^{p} .
\end{aligned}
$$

Here, $C_{4}=2 C_{3}$ if $A \geq 1$. We then take $\hat{\omega}_{k}=C_{4} \omega_{k}(k=0)$ and apply the assumption D) on any solution $v$ of (5.3) with $\hat{\eta}$ sufficiently small to find $\hat{A}, \hat{K}, \hat{L}$ depending on $\sup _{Q}|v|$, and therefore $M$, and construct the sequence $\left\{\hat{\omega}_{k}\right\}$ such that the relations in (5.1) hold and

$$
\oiiint_{\hat{Q}_{k}}\left|v-(v)_{k}\right|^{p} d z \leq \hat{\omega}_{k}^{p} \quad \forall k
$$

The new constants for $u$ will be chosen such that $K=\hat{K}, C_{4} A=\hat{A}$, with $\hat{A}$ being large and $A \geq 1$. The constants $L, \alpha_{0}$ will be determined later (using the constant $C_{4}, A$ ) so that $Q_{k+1} \subset \hat{Q}_{k+1}$ and $\omega_{k+1}=\max \left\{\eta \omega_{k}, L R_{k}^{\alpha_{0}}\right\}$. 

have

Choosing $K$ large (or equivalently $\hat{K}$ ) depending on $\eta$ such that, as $\omega_{k+1} \geq \eta \omega_{k}$, we

$$
\frac{4^{p}}{K^{p}} \omega_{k}^{p-2} \leq \omega_{k+1}^{p-2} \Leftrightarrow S_{k+1}^{2-p}\left(\frac{R}{K^{n+1}}\right)^{p} \leq S_{k}^{2-p}\left(\frac{R}{4 K^{n}}\right)^{p} \Rightarrow Q_{k+1} \subset \frac{1}{4} Q_{k} .
$$

Noting that $\frac{1}{4} Q_{k}$ is scaled by $R_{k}^{p}$ in the $t$ direction, we then deduce

$$
\begin{aligned}
\oiiint_{Q_{k+1}}|u-v|^{p} d z & \leq \frac{\omega_{k+1}^{p-2}}{\omega_{k}^{p-2}}(2 K)^{n+p} \oiiint_{\frac{1}{4} Q_{k}}|u-v|^{p} d z \leq \frac{\omega_{k+1}^{p-2}}{\omega_{k}^{p-2}}(2 K)^{n+p} \varepsilon R_{k}^{p} \int_{\frac{1}{2} Q_{k}}|D u|^{p} d z \\
& \leq C_{5} \varepsilon(2 K)^{n+p} \omega_{k+1}^{p-2} \omega_{k}^{2} .
\end{aligned}
$$

Here, we have used (5.5). Since $Q_{k+1} \subset \hat{Q}_{k+1}$, it follows that

$$
\begin{aligned}
\oiiint_{Q_{k+1}}\left|u-(u)_{k+1}\right|^{p} d z & \leq C_{6} \iint_{Q_{k+1}}|u-v|^{p} d z+C_{6} \int_{Q_{k+1}}\left|v-(v)_{k+1}\right|^{p} d z \\
& \leq C_{6} \iint_{Q_{k+1}}|u-v|^{p} d z+C_{6} \frac{\left|\hat{Q}_{k+1}\right|}{\left|Q_{k+1}\right|} \oiint_{\hat{Q}_{k+1}}\left|v-(v)_{k+1}\right|^{p} d z .
\end{aligned}
$$

The first term on the right is estimated as follows. We choose $\varepsilon$ small, depending on $K$ and thus $M$, such that for any given $\varepsilon^{\prime}>0$ we have via Young's inequality

$$
C_{5} \varepsilon(2 K)^{n+p} \omega_{k+1}^{p-2} \omega_{k}^{2} \leq \frac{1}{2} \omega_{k+1}^{p}+C_{7}\left(\varepsilon K^{n+p}\right)^{p / 2} \omega_{k}^{p} \leq \frac{1}{2} \omega_{k+1}^{p}+\varepsilon^{\prime} \omega_{k}^{p} .
$$

Meanwhile, since $v$ verifies $(5.2)$ in $\mathrm{D})$

$C_{6} \frac{\left|\hat{Q}_{k+1}\right|}{\left|Q_{k+1}\right|}\left|\int_{\hat{Q}_{k+1}}\right| v-\left.(v)_{k+1}\right|^{p} d z=C_{6} \frac{S_{k+1}^{p-2}}{\hat{S}_{k+1}^{p-2}} \int_{\hat{Q}_{k+1}}\left|v-(v)_{k+1}\right|^{p} d z \leq C_{8} \omega_{k+1}^{p-2} \hat{\omega}_{k+1}^{2} \leq \frac{1}{4} \omega_{k+1}^{p}$

if $\sqrt{C_{8}} \hat{\omega}_{k+1} \leq \omega_{k+1}$. Here, from the definition of $C_{4} A=\hat{A}, C_{8}=C_{6} C_{4}^{2-p}$ which depends only on $n$.

Recall that we also require $Q_{k+1} \subset \hat{Q}_{k+1}$. To this end, as $K=\hat{K}$, we need $S_{k+1}=$ $\frac{\omega_{k+1}}{A} \geq \frac{\hat{\omega}_{k+1}}{\hat{A}}=\hat{S}_{k+1}$ or $\omega_{k+1} \geq C_{4} \hat{\omega}_{k+1}$. This and the requirement $\leq \omega_{k+1} \geq \sqrt{C_{8}} \hat{\omega}_{k+1}$ are possible by choosing $L$ sufficiently large or $R_{0}$ is small with $\alpha_{0}<\hat{\alpha}_{0}$ and $\hat{\eta}$ small so that

$$
\omega_{k+1}=\max \left\{\eta \omega_{k}, L R^{\alpha_{0}}\right\} \geq \max \left\{\sqrt{C_{8}}, C_{4}\right\} \max \left\{\hat{\eta} \hat{\omega}_{k}, \hat{L} R^{\hat{\alpha}_{0}}\right\}=\max \left\{\sqrt{C_{8}}, C_{4}\right\} \hat{\omega}_{k+1} .
$$

We should note that once this requirement is fulfilled for $k=0$ then $\hat{\eta}$ is fixed and the above relation holds for all $k \geq 1$. By induction, we then define the sequence $\left\{\omega_{k}\right\}$ for $u$.

Hence,

$$
\oiiint_{Q_{k+1}}\left|u-(u)_{k+1}\right|^{p} d z \leq \frac{1}{2} \omega_{k+1}^{p}+\varepsilon^{\prime} \omega_{k}^{p}+\frac{1}{4} \omega_{k+1}^{p} \leq \omega_{k+1}^{p} \quad \text { for all } k \geq 0 .
$$

This shows that $u$ satisfies the same properties D) and $\mathcal{I}$ is open. In addition, an algebraic argument similar to [1, Proposition 3.1, p.44] applies to the sequence $\left\{\omega_{k}\right\}$ to get

$$
\oiiint_{Q_{k}}\left|u-(u)_{k}\right|^{p} d z \leq \omega_{k}^{p} \leq C R_{k}^{p \alpha_{0}} .
$$


Moreover, since $\omega_{k} \leq \omega_{0}$ and $p>2$, we have $Q_{R_{k}, S_{0}^{2-p} R_{k}^{p}} \subset Q_{k}$ and the above gives

$$
\oiiint_{Q_{R_{k}, S_{0}^{2-p} R_{k}^{p}}}\left|u-(u)_{k}\right|^{p} d z \leq C R_{k}^{\alpha_{0}} .
$$

By Campanato imbedding, the above implies that $u$ is Hölder continuous. Our proof is then complete.

\section{$6 \quad \mathcal{I}$ is closed}

In this section, we will show that the set $\mathcal{I}$ is closed in $[0,1]$. To proceed, we take a sequence $\left\{\nu_{k}\right\}$ in $\mathcal{I}$ such that $\nu_{k} \rightarrow \mu$ and show that $\mu \in \mathcal{I}$. Thus, let us consider a bounded weak solution to (2.1) with $\nu=\mu$. By II), there is a sequence of Hölder continuous weak solutions $v_{k}$ to

$$
v_{t}=\operatorname{div}\left(A\left(\nu_{k}, v, D v\right)\right) \text { in } Q_{1},
$$

such that $D v_{k}$ converges weakly in $L^{1}\left(Q_{1}\right)$ to $D u$. Moreover, the $L^{\infty}$ norms of $v_{k}$ 's are bounded uniformly in terms of that of $u$, and We will derive uniform estimates for various integral norms of $D v_{k}$ in terms of the $L^{\infty}$ norm of $u$. Once this is established, we obtain estimates for the derivatives of the limiting $u$ and its Hölder continuity to conclude that $\mu$ is in $\mathcal{I}$.

Let $v$ be any bounded weak solution to (6.1). We recall our assumptions here.

Let $\lambda_{\nu, v}, \Lambda_{\nu, v}$ be the ellipticity constants for the matrix $\left(A_{k l}^{i j}\right)=\frac{\partial A}{\partial \xi}(\nu, v, \xi)$, that is

$$
\sum_{i, j=1}^{m} \sum_{k, l=1}^{n} A_{k l}^{i j} \eta_{k}^{i} \eta_{l}^{j} \geq \lambda_{\nu, v}|\eta|^{2}, \quad \sum_{i, k}\left(\sum_{j, l} A_{k l}^{i j} \eta_{l}^{j}\right)^{2} \leq \Lambda_{\nu, v}^{2}|\eta|^{2}
$$

for any $\eta \in \mathbb{R}^{m n}$. Moreover, for some positive constants $\lambda_{\nu}, \Lambda_{\nu}$ we have

$$
\lambda_{\nu, v} \geq \lambda_{\nu}|D v|^{p-2}, \quad \Lambda_{\nu, v} \leq \Lambda_{\nu}|D v|^{p-2} .
$$

If $n>2$, we also assume that

$$
\frac{\Lambda_{\nu, v}}{\lambda_{\nu, v}}<\frac{n}{n-2}
$$

We also assume that there exists a positive constant $a_{\nu, v}$ such that

$$
\left|\frac{\partial A}{\partial v}(\nu, v, \xi)\right| \leq a_{\nu, v}|\xi|^{p-1} \text { with } 2 a_{\nu, v} M_{\nu, v}(p+n-1)<\sigma_{0} \widehat{\lambda}_{\nu}
$$

where $M_{\nu, v}=\sup _{Q_{\frac{2}{3}}}|v|$ and $\sigma_{0}$ is a fixed number in $(0,1)$ and

$$
\widehat{\lambda}_{\nu}=\left(1-\delta^{2}\right) \lambda_{\nu} \text { and } \delta=\frac{n-2}{n} \sup \left\{\frac{\Lambda_{\nu, v}}{\lambda_{\nu, v}}: \quad v \text { is a bounded solution }\right\} \text {. }
$$

Note that $\hat{\lambda}>0$ thanks to (6.3). 
Fixing $\nu$ in $I$ and a solution $v$ to (6.1), we will denote $a(v, \zeta)=A(\nu, v, \zeta)$ and also omit the parameter $\nu$ in the subscripts for $\lambda_{\nu, v}, \Lambda_{\nu, v}, a_{\nu, v}$ in the sequel.

The proof of Theorem 2.3 relies mainly on the following two lemmas which establish uniform bounds for the $L^{q}$ norms of $D v$. First of all, we need the following simple consequence of Sobolev's inequality. For any $q, r>0$, assuming $n>2$ as the case $n=2$ is easy, we have $q+r \frac{2}{n}=\frac{1}{2} q \frac{2 n}{n-2} \frac{n-2}{n}+r \frac{2}{n}$ and by Hölder and Sobolev's inequalities the following

$$
\begin{aligned}
\int_{\Omega}|V|^{q+r \frac{2}{n}} \phi^{2+\frac{4}{n}} d x & \leq\left(\int_{\Omega}\left[|V|^{\frac{1}{2} q} \phi\right]^{\frac{2 n}{n-2}} d x\right)^{\frac{n-2}{n}}\left(\int_{\Omega}|V|^{r} \phi^{2} d x\right)^{\frac{2}{n}} \\
& \leq C \int_{\Omega}\left|D\left(|V|^{\frac{1}{2} q} \phi\right)\right|^{2} d x\left(\int_{\Omega}|V|^{r} \phi^{2} d x\right)^{\frac{2}{n}} .
\end{aligned}
$$

Therefore, by integrating in $t$

$$
\iint_{Q_{1}}|V|^{q+r \frac{2}{n}} \phi^{2+\frac{4}{n}} d z \leq C \sup _{t \in(-1,0)}\left(\int_{\Omega}|V|^{r} \phi^{2} d x\right)^{\frac{2}{n}} \iint_{Q_{1}}\left|D\left(|V|^{\frac{1}{2} q} \phi\right)\right|^{2} d z .
$$

In the sequel, we will make use of difference quotients. For any vector valued function $f, i=1, \ldots, n$ and real number $h \neq 0$, we denote

$$
\delta_{h}^{(i)} f(x, t)=\frac{1}{h}\left(f\left(x+h e_{i}, t\right)-f(x, t)\right), \quad e_{i} \text { is the unit vector in the } i^{t h} \text { direction of } \mathbb{R}^{n} .
$$

If an argument holds for any $i$, we will simply omit the superscript $(i)$ in the above notation.

For any $v$ being a weak solution to a nice system, $v$ is Hölder continuous and the difference $\delta_{h} v$ weakly solves

$$
\left(\delta_{h} v\right)_{t}=\operatorname{div}\left(\delta_{h} a(v, D v)\right) .
$$

We first have the following estimate for "nice" solutions.

Lemma 6.1 Let $v$ be a Hölder continuous weak solution to (6.1). For any $\phi \in C_{0}^{1}\left(Q_{\frac{3}{4}}\right)$ there exists a constant $C$ depending on $M=\sup _{Q_{\frac{2}{3}}}|v|$ such that

$$
\sup _{t \in(-1,0)} \int_{\Omega}|D v|^{2} \phi^{2} d x+\lambda \iint_{Q_{1}}|D v|^{p-2}\left|D^{2} v\right|^{2} \phi^{2} d z \text { and } \iint_{Q_{1}}|D v|^{p+\frac{4}{n}} \phi^{2+\frac{4}{n}} d z \leq C \text {. }
$$

Proof: Let $\phi$ be in $C_{0}^{1}\left(Q_{\frac{2}{3}}\right)$. For any function $f$ in $(x, t), h \neq 0$ and $e=e_{i}(i=1, \ldots, n)$, we will write $\delta_{h}^{+} f(x, t)=(f(x+h e, t)-f(x, t)) / h$ and $\delta_{h}^{-} f(x, t)=(f(x, t)-f(x+h e, t)) / h$. Testing (6.6) with $\delta_{h}^{+} v \phi^{2}$ and integrating by part in $x$, we get

$$
\sup _{t \in(-1,0)} \int_{\Omega_{1}}\left|\delta_{h}^{+} v\right|^{2} \phi^{2} d x+\iint_{Q_{1}}\left\langle\delta_{h}^{+} a(v, D v), D\left(\delta_{h}^{+} v \phi^{2}\right)\right\rangle d z \leq \iint_{Q_{1}}\left|\delta_{h}^{+} v\right|^{2} \phi_{t} d z .
$$

We then set

$$
E_{0}=\left\{(x, t) \in Q_{1}:|D v(x, t)| \leq|D v(x+h, t)|\right\}, \quad E_{1}=Q_{1} \backslash E_{0} .
$$


We now split the integral of $\left\langle\delta_{h}^{+} a(v, D v), D\left(\delta_{h}^{+} v \phi^{2}\right)\right\rangle$ on $Q_{1}$ into those on $E_{0}, E_{1}$. On $E_{1}$, we have

$\left\langle\delta_{h}^{+} a(v, D v), D\left(\delta_{h}^{+} v \phi^{2}\right)\right\rangle=\left\langle\delta_{h}^{-} a(v, D v), D\left(\delta_{h}^{-} v \phi^{2}\right)\right\rangle=\left\langle\delta_{h}^{-} a(v, D v), D\left(\delta_{h}^{-} v\right) \phi^{2}+\delta_{h}^{-} v D\left(\phi^{2}\right)\right\rangle$.

Concerning the term $\delta_{h}^{-} a(v, D v)$, we write

$$
\begin{aligned}
\delta_{h}^{-} a(v, D v)= & \frac{1}{h}[a(v(x, t), D v(x, t))-a(v(x, t), D v(x+h, t))]+ \\
& \frac{1}{h}[a(v(x, t), D v(x+h, t))-a(v(x+h, t), D v(x+h, t))] \\
= & \int_{0}^{1} \frac{\partial a}{\partial \xi}(v, s D v(x, t)+(1-s) D v(x+h, t)) D \delta_{h}^{-} v d s+ \\
& \int_{0}^{1} \frac{\partial a}{\partial v}(s v(x, t)+(1-s) v(x+h, t), D v(x+h, t)) \delta_{h}^{-} v d s .
\end{aligned}
$$

Using the fact that $|s D v(x, t)+(1-s) D v(x+h, t)| \geq|D v(x+h, t)|$ on $E_{1}$ and the ellipticity condition of $\partial a / \partial \xi$ we get

$$
\left\langle\frac{\partial a}{\partial \xi}(v, s D v(x, t)+(1-s) D v(x+h, t)) D \delta_{h}^{-} v d s, D \delta_{h}^{-} v\right\rangle \geq \lambda|D v(x+h, t)|^{p-2}\left|D \delta_{h}^{-} v\right|^{2} .
$$

This term will stay on the left of (6.8). On the other hand, bty (6.4), we have

$$
\left|\frac{\partial a}{\partial v}(s v(x, t)+(1-s) v(x+h, t), D v(x+h, t)) \delta_{h}^{-} v\right| \leq\left|a_{v}\right||D v(x+h, t)|^{p-1}\left|\delta_{h}^{-} v\right| .
$$

Thus, by Young's inequality, we have for any positive $\varepsilon$ the following

$$
\begin{aligned}
\mid\left\langle\frac{\partial a}{\partial v}(s v(x, t)\right. & \left.+(1-s) v(x+h, t), D v(x+h, t)) \delta_{h}^{-} v, D\left(\delta_{h}^{-} v\right)\right\rangle \mid \leq \\
& \leq \varepsilon|D v(x+h, t)|^{p-2}\left|D\left(\delta_{h}^{-} v\right)\right|^{2}+C(\varepsilon)\left|a_{v}\right|^{2}|D v(x+h, t)|^{p}\left|\delta_{h}^{-} v\right|^{2} .
\end{aligned}
$$

The above term will be on the right hand side of (6.8).

Similar argument will apply to the set $E_{0}$. We then choose $\varepsilon$ sufficiently small and derive from (6.8) and the above estimates

$$
\begin{array}{r}
\sup _{t \in(-1,0)} \int_{\Omega_{1}}\left|\delta_{h} v\right|^{2} \phi^{2} d x+\frac{\lambda}{2} \iint_{Q_{1}}\left|v_{d}\right|^{p-2}\left(\left|D\left(\delta_{h}^{+} v\right)\right|^{2}+\left|D \delta_{h}^{-} v\right|^{2}\right) \phi^{2} d z \leq \\
\iint_{Q_{1}} \frac{\left|a_{v}\right|^{2}}{\lambda}\left|v_{D}\right|^{p}\left|V_{h}\right|^{2} \phi^{2}+\left|v_{D}\right|^{2}\left(\left|\phi_{t}\right|+\Lambda\left|v_{D}\right|^{p-2}|D \phi|^{2}+1\right) d z,
\end{array}
$$

where $v_{D}=\max \{|D v(x, t)|,|D v(x+h, t)|\}, v_{d}=\min \{|D v(x, t)|,|D v(x+h, t)|\}$ and $V_{h}=$ $\max \left\{\left|\delta_{h}^{+} v\right|,\left|\delta_{h}^{-} v\right|\right\}$.

Sending $h$ to zero, we get

$$
\begin{aligned}
& \sup _{t \in(-1,0)} \int_{\Omega_{1}}|D v|^{2} \phi^{2} d x+\frac{\lambda}{2} \iint_{Q_{1}}|D v|^{p-2}\left|D^{2} v\right|^{2} \phi^{2} d z \leq \\
& \iint_{Q_{1}} \frac{\left|a_{v}\right|^{2}}{\lambda}|D v|^{p+2} \phi^{2}+|D v|^{2}\left(\left|\phi_{t}\right|+\Lambda|D v|^{p-2}|D \phi|^{2}+1\right) d z .
\end{aligned}
$$

Of course the above argument is justified if $D v \in L_{l o c}^{p+2}$. This fact will be proven in Lemma 6.2 following this proof. 
We now estimate the integral of $|D v|^{p+2}$ in (6.10). By integrating by parts in $x$

$\iint_{Q_{1}}|D v|^{p+2} \phi^{2} d z=\iint_{Q_{1}} v D\left(|D v|^{p+1} \phi^{2}\right) d z \leq M \iint_{Q_{1}}\left(\left|D^{2} v\right||D v|^{p} \phi^{2}+|D v|^{p+1} \phi|D \phi|\right) d z$,

where $M=M_{\nu, v}=\sup _{Q_{\frac{3}{4}}}|v|$.

Young's inequality applying to the right then gives

$\iint_{Q_{1}}|D v|^{p+2} \phi^{2} d z \leq \varepsilon \iint_{Q_{1}}|D v|^{p+2} \phi^{2} d z+C(\varepsilon) M^{2} \iint_{Q_{1}}\left(\left|D^{2} v\right|^{2}|D v|^{p-2} \phi^{2}+|D v|^{p}|D \phi|^{2}\right) d z$.

Thus, for $\varepsilon=\frac{1}{2}$, we obtain

$$
\frac{a_{v}^{2}}{\lambda} \iint_{Q_{1}}|D v|^{p+2} \phi^{2} d z \leq 4 \frac{a_{v}^{2} M^{2}}{\lambda} \iint_{Q_{1}}|D v|^{p-2}\left|D^{2} v\right|^{2} \phi^{2}+|D v|^{p}|D \phi|^{2} d z .
$$

Using this in (6.10) and the assumption on the smallness of $a_{v} M$ in (6.4), we obtain

$$
\begin{gathered}
\sup _{t \in(-1,0)} \int_{\Omega}|D v|^{2} \phi^{2} d x+\lambda \iint_{Q_{1}}|D v|^{p-2}\left|D^{2} v\right|^{2} \phi^{2} d z \leq \\
C \iint_{Q_{1}}|D v|^{2}\left(\left|\phi_{t}\right|+\left(|D v|^{p-2} \Lambda+\lambda\right)|D \phi|^{2}+1\right) d z .
\end{gathered}
$$

By Caccioppoli's inequality we note that

$$
\iint_{Q_{1}}|D v|^{2}\left(\left|\phi_{t}\right|+|D v|^{p-2}|D \phi|^{2}\right) d z \leq C\left(\left|\phi_{t}\right|,|D \phi|\right) \iint_{Q_{1}}|v|^{2} d z \leq C(M) .
$$

The above and (6.11) then imply

$$
\sup _{t \in(-1,0)} \int_{\Omega_{1}}|D v|^{2} \phi^{2} d x+\iint_{Q_{1}}|D v|^{p-2}\left|D^{2} v\right|^{2} \phi^{2} d z \leq C(M) .
$$

We now make use of (6.5), with $V=D v$ and $q=p, r=2$, and combine with the above to get the second estimate in (6.7) and complete the proof.

To justify the calculation leading to (6.10), we now show that

Lemma 6.2 Spatial derivatives of Hölder continuous weak solutions $v$ to (6.1) are in $L_{l o c}^{p+2}$.

Proof: Let $v$ be a Hölder continuous weak solutions to (6.1). We will show that at almost every point $z_{0}=\left(x_{0}, t_{0}\right) \in Q_{1}$ with $D v\left(z_{0}\right) \neq 0$ and $R$ is sufficiently small then there is a constant $C$ such that

$$
\iint_{Q_{R}}\left|\delta_{h_{k}} v\right|^{p+2} d z \leq C R^{-2} \iint_{Q_{4 R}}|D v|^{p} d z \quad \text { for some sequence } h_{k} \rightarrow 0 \text {. }
$$

Here, $Q_{R}=B_{R}\left(x_{0}\right) \times\left(t_{0}-R^{2}, t_{0}\right)$ and $Q_{R} \subset Q_{4 R} \subset Q_{1}$. If this is not true then there will be a sequence $R_{k} \rightarrow 0$ such that

$$
\iint_{Q_{R_{k}}}\left|\delta_{h_{k}} v\right|^{p+2} d z>k R_{k}^{-2} \iint_{Q_{4 R_{k}}}|D v|^{p} d z \quad \text { for any sequence } h_{k} \rightarrow 0
$$


We then choose $h_{k}=h R_{k}$ for some sufficiently small and positive $h$ to be determined later. By scaling, with $v_{k}(X, T)=v\left(x_{0}+R_{k} X, t_{0}+R_{k}^{2} T\right)$, we get a sequence of functions $v_{k}$ on $Q_{1}$ such that

$$
\iint_{Q_{\frac{1}{4}}}\left|\delta_{h} v_{k}\right|^{p+2} d z \geq k \iint_{Q_{1}}\left|D v_{k}\right|^{p} d z
$$

Since $v$ is Hölder continuous and $R_{k} \rightarrow 0$ we see that $v_{k}$ can be arbitrarily close to $\left(v_{k}\right)_{Q_{1}}$ on $Q_{1}$. Thus, $v_{k}$ approximately solves the following system

$$
U_{t}=\operatorname{div}\left(a\left(\left(v_{k}\right)_{Q_{1}}, D U\right)\right) \text {. }
$$

For such system, which does not explicitly depend on $U$, we can find (see Remark 6.4 at the end of this section) a function $C(x)$ which is bounded if $x$ is bounded such that

$$
\iint_{Q_{\frac{1}{4}}}|D U|^{p+2} d z \leq C\left(\iint_{Q_{\frac{1}{2}}}|D U|^{p} d z\right) .
$$

For sufficiently small $h>0$, the above yields

$$
\iint_{Q_{\frac{1}{4}}}\left|\delta_{h} U\right|^{p+2} d z \leq C\left(\iint_{Q_{\frac{1}{2}}}|D U|^{p} d z\right) .
$$

Our approximation results then give a sequence $\left\{U_{k}\right\}$ of weak solutions to (6.15) satisfying

$$
\iint_{Q_{\frac{1}{2}}}\left|D U_{k}\right|^{p} d z \leq c \iint_{Q_{\frac{1}{2}}}\left|D v_{k}\right|^{p} d z
$$

and $U_{k}-v_{k} \rightarrow 0$ in $L^{p}\left(Q_{\frac{1}{4}}\right)$ (as well as in $L^{q}\left(Q_{\frac{1}{4}}\right)$ for any $q>1$ because $U_{k}, v_{k}$ are bounded). Moreover, $D U_{k}-D v_{k} \rightarrow 0$ weakly in $L^{p}\left(Q_{\frac{1}{4}}\right)$.

Together, we have the following estimates

$$
k \iint_{Q_{1}}\left|D v_{k}\right|^{p} d z \leq \iint_{Q_{\frac{1}{4}}}\left|\delta_{h} v_{k}\right|^{p+2} d z, \quad \iint_{Q_{\frac{1}{4}}}\left|\delta_{h} U_{k}\right|^{p+2} d z \leq C\left(\iint_{Q_{\frac{1}{2}}}\left|D v_{k}\right|^{p} d z\right) .
$$

With $h$ being fixed and $U_{k}, v_{k}$ being bounded, we have $\delta_{h}\left(U_{k}\right)-\delta_{h}\left(v_{k}\right) \rightarrow 0$ in $L^{q}$ for all $q>1$ as $k \rightarrow \infty$. Moreover, since $\iint_{Q_{\frac{1}{2}}}\left|D v_{k}\right|^{p} d z \rightarrow D v\left(z_{0}\right) \neq 0$, the above gives a contradiction when $k \rightarrow \infty$. Thus, (6.13) holds almost everywhere on the set where $D v \neq 0$. Finally, by sending $h$ to 0 it is easy to see that (6.13) implies $D v \in L_{l o c}^{p+2}$.

To get estimates for higher powers of $|D v|$, we need the following lemma.

Lemma 6.3 Let $v$ be a Hölder continuous weak solution to (6.1) and $\alpha$ be a positive number. Assume that $\frac{\alpha}{2+\alpha}=\delta_{\alpha, v} \frac{\lambda_{v}}{\Lambda_{v}}$ for some $\delta_{\alpha, v} \in(0,1)$ and

$$
2 a_{v} M(p+\alpha+1)<\sigma_{0} \widehat{\lambda}_{v}, \quad \text { with } \widehat{\lambda}_{v}=\left(1-\delta_{\alpha, v}^{2}\right) \lambda_{v}, \quad \sigma_{0} \in(0,1) .
$$


If $\phi \in C_{0}^{1}\left(Q_{\frac{3}{4}}\right)$ then

$$
\iint_{Q_{1}}|D v|^{p+\alpha+(2+\alpha) \frac{2}{n}} \phi^{2+\frac{4}{n}} d z \leq C\left(\iint_{Q_{1}}|D v|^{2+\alpha}\left(\left|\phi_{t}\right|+|D v|^{p-2}|D \phi|^{2}+1\right) d z\right) .
$$

Proof: To proceed, we recall the following facts in [7]. From the ellipticity condition of $\frac{\partial a}{\partial \xi}=\left(A_{k l}^{i j}\right)$ we have for $\kappa_{v}=\lambda_{v} / \Lambda_{v}^{2}$ and $\nu_{v}=\lambda_{v} / \Lambda_{v}$ that

$$
\sum_{i, k}\left(\eta_{i}^{k}-\kappa A_{k l}^{i j} \eta_{l}^{j}\right)^{2} \leq\left(1-2 \kappa_{v} \lambda_{v}+\kappa_{v}^{2} \Lambda_{v}^{2}\right)|\eta|^{2}=\left(1-\nu_{v}^{2}\right)|\eta|^{2}
$$

The lemma [7, p.677] then gives $D \zeta D\left(\zeta|\zeta|^{\alpha}\right) \geq \mu^{\frac{1}{2}}(\alpha)|D \zeta|\left|D\left(\zeta|\zeta|^{\alpha}\right)\right|$ for any $\zeta: \mathbb{R}^{n} \rightarrow$ $\mathbb{R}^{n m}$ and $\mu(\alpha)=1-\left(\frac{\alpha}{2+\alpha}\right)^{2}$. Therefore, with $\zeta=D v$

$$
\begin{aligned}
\sum \frac{\partial a}{\partial \xi} D^{2} v D\left(D v|D v|^{\alpha}\right) & =\frac{1}{\kappa_{v}}\left[\kappa_{v} \sum\left(\frac{\partial a}{\partial \xi} D^{2} v-D^{2} v\right) D\left(D v|D v|^{\alpha}\right)\right]+D(D v) D\left(D v|D v|^{\alpha}\right) \\
& \geq \frac{1}{\kappa_{v}}\left(\mu^{\frac{1}{2}}(\alpha)-\left(1-\nu_{v}^{2}\right)^{\frac{1}{2}}\right)|D(D v)|\left|D\left(D v|D v|^{\alpha}\right)\right| .
\end{aligned}
$$

Thus, if $\frac{\alpha}{2+\alpha}=\delta_{\alpha, v} \frac{\lambda_{v}}{\Lambda_{v}}$ for some $\delta_{\alpha, v} \in(0,1)$ then the constant in the right hand side is

$$
\frac{1}{\kappa_{v}}\left(\mu(\alpha)^{\frac{1}{2}}-\left(1-\nu_{v}^{2}\right)^{\frac{1}{2}}\right)=\frac{1}{\kappa_{v}} \frac{\nu_{v}^{2}-\frac{\alpha^{2}}{(2+\alpha)^{2}}}{\left(\mu(\alpha)^{\frac{1}{2}}+\left(1-\nu_{v}^{2}\right)^{\frac{1}{2}}\right)} \geq\left(1-\delta_{\alpha, v}^{2}\right) \frac{\nu_{v}^{2}}{\kappa_{v}}=\left(1-\delta_{\alpha, v}^{2}\right) \lambda_{v} .
$$

Hence, by the assumption on $\lambda_{v}$, we get

$$
\begin{aligned}
\sum \frac{\partial a}{\partial \xi} D^{2} v D\left(D v|D v|^{\alpha}\right) & \geq \frac{1}{\kappa_{v}}\left(\mu^{\frac{1}{2}}(\alpha)-\left(1-\nu_{v}^{2}\right)^{\frac{1}{2}}\right)|D(D v)| \mid D\left(D v|D v|^{\alpha}\right) \\
& \geq\left(1-\delta_{\alpha, v}^{2}\right) \lambda_{v}|D v|^{\alpha}\left|D^{2} v\right|^{2}=\widehat{\lambda}_{v}|D v|^{p-2+\alpha}\left|D^{2} v\right|^{2}
\end{aligned}
$$

The following calculation will be rigorously justified by using difference quotient operator $\delta_{h}$, as in the previous lemmas, in place of the differentiation $D$ below. However, in order to be more suggestive, we will write (6.6) formally as

$$
(D v)_{t}=\operatorname{div}\left(\frac{\partial a}{\partial \xi}(v, D v) D^{2} v+\frac{\partial a}{\partial v}(v, D v) D v\right)
$$

Testing (6.20) with $D v|D v|^{\alpha} \phi^{2}$ to obtain (compare with (6.9))

$$
\begin{aligned}
\sup _{t} \int_{\Omega_{1}} & |D v|^{2+\alpha} \phi^{2} d x+\iint_{Q_{1}}\left\langle\frac{\partial a}{\partial \xi}(v, D v) D^{2} v, D\left(D v|D v|^{\alpha}\right) \phi^{2}\right\rangle d z \leq \iint_{Q_{1}}|D v|^{2+\alpha}\left|\phi_{t}\right| d z \\
& +\iint_{Q_{1}}\left|\left\langle\frac{\partial a}{\partial \xi}(v, D v) D^{2} v, D v|D v|^{\alpha} D \phi \phi\right\rangle\right|+\left|\frac{\partial a}{\partial v}\right|\left(|D v|^{2+\alpha} \phi^{2}+|D v|^{1+\alpha}|\phi D \phi|\right) d z .
\end{aligned}
$$

Using (6.19) and Young's inequality, we deduce

$$
\begin{aligned}
& \sup _{t} \int_{\Omega_{1}}|D v|^{2+\alpha} \phi^{2} d x+\widehat{\lambda}_{v} \iint_{Q_{1}}\left|D^{2} v\right|^{2}|D v|^{p-2+\alpha} \phi^{2} d z \leq \\
& \frac{\left|a_{v}\right|^{2}}{\widehat{\lambda}_{v}} \iint_{Q_{1}}|D v|^{p+2+\alpha} \phi^{2} d z+C \iint_{Q_{1}}|D v|^{2+\alpha}\left(\left|\phi_{t}\right|+|D v|^{p-2}|D \phi|^{2}+1\right) d z .
\end{aligned}
$$


Again, since $v$ is Hölder continous, similar argument as that of Lemma 6.2 shows that $D v \in L_{l o c}^{p+2+\alpha}$ and justifies the above and below calculation. We now estimate the integral of $|D v|^{p+2+\alpha} \phi^{2}$. By integrating by parts in $x$, we have

$$
\begin{aligned}
& \iint_{Q_{1}}|D v|^{p+2+\alpha} \phi^{2} d z=\iint_{Q_{1}} v D\left(D v|D v|^{p+\alpha} \phi^{2}\right) d z \\
& \leq M \iint_{Q_{1}}(p+\alpha+1)\left|D^{2} v\right||D v|^{p+\alpha} \phi^{2}+|D v|^{p+1+\alpha} \phi|D \phi| d z
\end{aligned}
$$

where $M=\sup _{Q_{\frac{2}{3}}}|v|$. Young's inequality applying to the right then gives

$$
\begin{aligned}
& \iint_{Q_{1}}|D v|^{p+2+\alpha} \phi^{2} d z \leq\left(\frac{1}{2}+\varepsilon\right) \iint_{Q_{1}}|D v|^{p+2+\alpha} \phi^{2} d z+ \\
& \quad[2 M(p+\alpha+1)]^{2} \iint_{Q_{1}}\left|D^{2} v\right|^{2}|D v|^{p-2+\alpha} \phi^{2} d z+C(\varepsilon) M^{2} \iint_{Q_{1}}|D v|^{p+\alpha}|D \phi|^{2} d z .
\end{aligned}
$$

We choose $\varepsilon<1 / 2$ in the above to obtain an estimate for the integral of $|D v|^{p+2+\alpha} \phi^{2}$. Using this in (6.21) and the assumption (6.17) on $\left|a_{v} M\right|$ and $\hat{\lambda}_{v}$, we obtain

$$
\begin{array}{r}
\sup _{t} \int_{\Omega_{1}}|D v|^{2+\alpha} \phi^{2} d x+\left(1-\sigma_{0}^{2}\right) \widehat{\lambda}_{v} \iint_{Q_{1}}\left|D^{2} v\right|^{2}|D v|^{p-2+\alpha} \phi^{2} d z \leq \\
C \iint_{Q_{1}}|D v|^{2+\alpha}\left(\left|\phi_{t}\right|+\widehat{\lambda}|D v|^{p-2}|D \phi|^{2}+1\right) d z .
\end{array}
$$

The above also gives similar estimate for $\left\||D v|^{(p+\alpha) / 2} \phi\right\|_{V\left(Q_{1}\right)}$. Applying (6.5), with $V=D v$ and $q=p+\alpha, r=2+\alpha$, we get the lemma.

We are now ready to give

Proof of Theorem 2.3: By M.2) (see (6.4)), we can choose a number $\beta>n-2$ and some $\delta^{\prime} \in(0,1)$ such that for $M=\sup _{Q_{\frac{3}{4}}}|v|$

$$
2 a_{v} M(p+\beta+1)<\sigma_{0} \widehat{\lambda}_{v} \text {, and } \frac{\beta}{\beta+2}=\delta^{\prime} \frac{\lambda_{v}}{\Lambda_{v}}>\frac{n-2}{n} .
$$

Clearly, starting with $\alpha_{0} \leq 2$ (thus $p+\alpha_{0} \leq p+2$ ), we can find finitely many numbers $\alpha_{0}, \ldots, \alpha_{K}$ such that

$$
2 a_{v} M\left(p+\alpha_{k}+1\right)<\sigma_{0} \widehat{\lambda} \quad \text { and } \quad \frac{\alpha_{k}}{\alpha_{k}+2}=\delta_{s, v}^{(k)} \frac{\lambda_{v}}{\Lambda_{v}}, \quad \delta_{s, v}^{(k)} \leq \delta^{\prime}
$$

for any $k \leq K$. Moreover, it is easy to see that we can also choose $\delta_{s, v}^{(k)}$ such that $\alpha_{k} \leq$ $\alpha_{k+1} \leq \alpha_{k}+\left(2+\alpha_{k}\right) \frac{2}{n}, \alpha_{K}=\beta$ and $p+\alpha_{k+1} \leq p+\alpha_{k}$.

Since $Q_{\frac{5}{7}} \subset Q_{\frac{3}{4}}$, by the Caccioppoli inequality we obtain $\|D u\|_{L^{p}\left(Q_{\frac{5}{7}}\right)} \leq C(M)$. Using the estimate for $|D v|^{p+2}$ in Lemma 6.1 and a cut-off function $\phi$ for $Q_{\frac{2}{3}}, Q_{\frac{5}{7}}$ in Lemma 6.3. we see that

$$
\iint_{Q_{\frac{2}{3}}}|D v|^{p+2} d z \leq C(M) .
$$


Let the function $\phi$ in Lemma 6.3 be the cut-off functions for $Q_{R_{k}}$ and $Q_{R_{k-1}}$ with $R_{k}=\frac{2}{3}-k \frac{1}{6 K}, k=1, \ldots, K$. By induction, the choice of $\alpha_{k}$ allows us to obtain the following estimate from (6.18)

$$
\iint_{Q_{\frac{1}{2}}}|D v|^{q_{K}} d z \leq C(K, M), \quad q_{K}=p+\alpha_{K}+\left(2+\alpha_{K}\right) \frac{2}{n} .
$$

Note that $q_{K}>n+p$ because $\alpha_{K}=\beta>n-2$.

Now, let $u$ be a weak solution which is, by II), approximated by a sequence $\left\{v_{k}\right\}$ of weak solutions to nice systems and $D v_{k} \rightarrow D u$ weakly in $L^{1}\left(Q_{\frac{1}{2}}\right)$. By the semicontinuity of seminorms and (6.23), we have for any $Q_{R} \subset Q_{\frac{1}{2}}$ that

$$
\iint_{Q_{R}}|D u|^{q_{K}} d z \leq \liminf _{k \rightarrow \infty} \iint_{Q_{R}}\left|D v_{k}\right|^{q_{K}} d z \leq C(M) .
$$

Hence, with $q=q_{K} / p$

$$
\iint_{Q_{R}}|D u|^{p} d z \leq\left(\iint_{Q_{R}}|D u|^{q_{K}} d z\right)^{\frac{1}{q}}\left|Q_{R}\right|^{1-\frac{1}{q}} \leq C(M) R^{n+p-(n+p) \frac{1}{q}}=C(M) R^{n+\alpha} .
$$

Here, $\alpha=p-(n+p) \frac{1}{q}=\frac{p}{q_{K}}\left(q_{K}-n-p\right)$ is positive. Hölder continuity for $u$ then follows from the above estimate and the Poincaré inequality in Lemma 3.1. Thus, $u$ can be approximated by solutions to systems that do not explicitly depend on $u$ and satisfy the property $\mathrm{D}$ ). This implies that $u$ satisfies $\mathrm{D}$ ) and $\mathcal{I}$ is closed.

Remark 6.4 We should note that the estimate (6.16) for systems with coefficients independent of their solutions could be derived directly from our proof. Indeed, for such systems $\frac{\partial a}{\partial v}=0$. Therefore, our arguments which lead to (6.10) and (6.21) would not yield the integral of $|D u|^{p+2+\alpha}$ on the right hand sides, and give a similar bound as in (6.16) for the integral of $|D U|^{q_{K}}$ for some $q_{K}>n+p$. Hence, the calculation in our lemmas is justified.

\section{References}

[1] E. DiBenedetto Degenerate Parabolic Equations. Springer-Verlag Universitext, 1993.

[2] N. Dunford and J.T. Schwartz Linear Operator - Part I. Pure and Appl. Math, J. Wiley, New York, 1993.

[3] M. Giaquinta and M. Struwe. On the partial regularity of weak solutions of nonlinear parabolic systems. Math. Z. Vol. 179 (1982), pp. 437-451.

[4] E. Giusti Direct Methods in the Calculus of Variations. World Scientific, 2003.

[5] O. John and J. Stara. Some (new) counterexamples of parabolic systems. Commentat math. Univ. Carol., 36 (1995), pp. 503-510.

[6] O. John and J. Stara. On the regularity of weak solutions to parabolic systems in two spatial dimensions. Comm. P.D.E., 27(1998), pp. 1159-1170. 
[7] E. Kalita. On Holder continuity of solutions of nonlinear parabolic systems. Comment. Math. Carolinae., 35,4(1994), pp. 675-680.

[8] J. Kinnunen and J.L. Lewis Higher integrability for parabolic systems of $p$-Laplacian type Duke Math. J. Vo.102, No. 2 (2000), pp 253-271.

[9] O. A. Ladyzenskaja, V. A. Solonnikov, and N. N. Ural'tseva. Linear and Quasilinear Equations of Parabolic Type. AMS Transl. Monographs, vol. 23, 1968.

[10] D. Le. Partial regularity of solutions to a class of strongly coupled degenerate parabolic systems. Discrete Contin. Dyn. Syst. 2005, suppl., pp. 576-586.

[11] D. Le. Global existence for a class of strongly coupled parabolic systems. Ann. Mat. Pura Appl. (4) 185 (2006), no. 1, pp. 133-154.

[12] D. Le Regularity of BMO Weak Solutions to Nonlinear Parabolic Systems via Homotopy. to appear on Trans. Amer. Math. Soc.

[13] D. Le and T. Nguyen, Everywhere regularity of solutions to a class of strongly coupled degenerate parabolic systems. Comm. Partial Differential Equations 31 (2006), no. 1-3, pp. 307-324.

[14] D. Le and T. Nguyen. Global existence for a class of triangular parabolic systems on domains of arbitrary dimension. Proc. Amer. Math. Soc. 133 (2005), no. 7, pp. 1985-1992.

[15] G. M. Lieberman Second Order Parbolic Differential Equations. World Scientific, 1998.

[16] J. L. Lions Quelques methodes de resolution des problemes aus limites non-lineaires. Dunod, Paris (1969). 